\title{
High-resolution spectroscopy of the blue compact dwarf galaxy Haro 15 - II. Chemodynamics
}

\author{
Guillermo F. Hägele, ${ }^{1,2,3 \star}$ Verónica Firpo, ${ }^{1,2}$ Guillermo Bosch, ${ }^{1,2}$ Ángeles I. Díaz ${ }^{3}$ \\ and Nidia Morrell ${ }^{4}$ \\ ${ }^{1}$ Facultad de Ciencias Astronómicas y Geofísicas, Universidad Nacional de la La Plata, Paseo del Bosque s/n, 1900 La Plata, Argentina \\ ${ }^{2}$ IALP-Conicet, Paseo del Bosque s/n, 1900 La Plata, Argentina \\ ${ }^{3}$ Departamento de Física Teórica, C-XI, Universidad Autónoma de Madrid, 28049 Madrid, Spain \\ ${ }^{4}$ Las Campanas Observatory, Carnegie Observatories, Casilla 601, La Serena, Chile
}

Accepted 2012 March 1. Received 2012 February 17; in original form 2011 November 11

\begin{abstract}
We present a detailed study of the physical properties of the nebular material in four starforming knots of the blue compact dwarf galaxy Haro 15. Using long-slit and echelle spectroscopy obtained at Las Campanas Observatory, we study the physical conditions (electron density and temperatures), ionic and total chemical abundances of several atoms, reddening and ionization structure, for the global flux and for the different kinematical components. The latter was derived by comparing the oxygen and sulphur ionic ratios to their corresponding observed emission-line ratios (the $\eta$ and $\eta^{\prime}$ plots) in different regions of the galaxy. Applying the direct method or empirical relationships for abundance determination, we perform a comparative analysis between these regions. The similarities found in the ionization structure of the different kinematical components imply that the effective temperatures of the ionizing radiation fields are very similar in spite of some small differences in the ionization state of the different elements. Therefore, the different gaseous kinematical components identified in each star-forming knot are probably ionized by the same star cluster. However, the difference in the ionizing structure of the two knots with knot A showing a lower effective temperature than knot B suggests a different evolutionary stage for them consistent with the presence of an older and more evolved stellar population in the first.
\end{abstract}

Key words: ISM: abundances - H II regions - galaxies: abundances - galaxies: fundamental parameters - galaxies: individual: Haro 15 - galaxies: starburst.

\section{INTRODUCTION}

Giant extragalactic $\mathrm{H}_{\text {II }}$ regions (GHIIRs) are extended luminous objects, which are observed on the discs of spirals and in irregular galaxies. GHIIRs are formed due to the presence of a large number of young and massive stars whose ultraviolet (UV) flux ionizes the surrounding gas. The behaviour of emission lines observed in $\mathrm{H}_{\mathrm{II}}$ galaxies and blue compact dwarf (BCD) spectra resembles those of giant $\mathrm{H}_{\mathrm{II}}$ regions. We can, therefore, use the same analysis techniques as developed for the latter to derive the temperatures, densities and chemical composition of the interstellar gas in the star formation bursts detected in these low-metallicity galaxies (Sargent $\&$ Searle 1970; French 1980; Terlevich et al. 1991). BCD galaxies present strong starbursts easily identified through their strong and narrow emission lines, low-metallicity environments and a complex

${ }^{\star}$ E-mail: ghagele@ fcaglp.unlp.edu.ar history of star formation. These facts make them interesting objects to study metallicity effects in galaxies (Kunth \& Östlin 2000).

As mentioned in Firpo et al. (2011, hereafter Paper I), Haro 15 has an absolute magnitude $M_{B}=-20.69$, a surface brightness $\mu=$ $18.56 \mathrm{mag} \mathrm{arcsec}^{-2}$ and a colour $B-V=0.33$ (Cairós et al. 2001). At a distance of $86.6 \mathrm{Mpc}$ (de Vaucouleurs et al. 1991), Haro 15 meets the criteria for a luminous compact blue galaxy (Hoyos et al. 2004), although some authors suggest that it may represent the final outcome of a merger between a dwarf elliptical and a gas-rich dwarf galaxy or HI cloud (Östlin 1998; Cumming et al. 2008). The interactions taking place during the merging process would act as the starburst trigger.

Shi et al. (2005) derived an overall average oxygen abundance of $12+\log (\mathrm{O} / \mathrm{H})=8.33$ for the Haro 15 galaxy, based on an electron temperature determined to be $8330 \mathrm{~K}$. It was, however, previously known from $\mathrm{H} \alpha$ imaging (Cairós et al. 2001) that the galaxy shows a complex morphology, where the star-forming region can be split among a large number of knots scattered throughout the whole 
galaxy. López-Sánchez \& Esteban (2009b) obtained spectroscopic information from individual regions and determined densities, temperatures and chemical abundances for several knots within Haro 15. Oxygen abundances were derived for regions A and B (names following Cairós et al. 2001) and found to be $8.37 \pm 0.10$ and $8.10 \pm$ 0.06 , respectively.

Esteban \& Vílchez (1992) introduced the term 'chemodynamics' after merging chemical and kinematical information in their analysis of the Wolf-Rayet ring nebula NGC 6888. In their research, these authors combined high-resolution spectra with observations at high spatial resolution for different areas of the nebula, which enabled them to analyse the different velocity components of the studied region.

James et al. (2009) studied the BCD galaxy Mrk 996 using high spectral resolution data obtained with VLT VIMOS in its integral field unit (IFU) mode. They found that this galaxy shows multiple components in its emission-line profiles, evident as relatively broad and narrow components, and were able to deblend them in order to analyse their physical properties separately. According to the authors this peculiar BCD galaxy has extremely dense gas in its nuclear region, where stellar outflows and shock fronts contribute to generating the relatively broad feature. However, James and collaborators could not find evidence of complex emission-line profiles for the auroral lines sensitive to temperature. The [O III] $\lambda 4363$ and [N II] $\lambda 5755$ emission lines, present in their spectra, showed simple profiles which could be fitted by a broad Gaussian function.

In a previous work we presented a detailed study of the internal kinematics of the nebular material in multiple knots of the BCD galaxy Haro 15 (Paper I) from echelle spectroscopy. In that paper we have performed a thorough analysis of its emission lines, including multiple-component fits to the profiles of their strong emission lines. Our results have shown that the giant $\mathrm{H}$ II regions of Haro 15 present a complex structure in all their emission profiles, detected both in recombination and forbidden lines. The emission lines of the brightest region, Haro $15 \mathrm{~A}$, can be split into at least two strong narrow components plus a broad one. Although the observed regions tend to follow the galaxy kinematics, the components of knot A have relative velocities that are too large to be explained by galactic rotation. Almost all knots follow the relation between luminosity and velocity dispersion found for virialized systems, when considering either single profile fittings or the strong narrow components in more complex fits. The one-component fits show a relatively flatter slope.

Generally, the flux calibration of echelle data is difficult to check and the weak auroral emission lines do not have enough $\mathrm{S} / \mathrm{N}$ to allow the accurate measurement necessary for a reliable estimate of the temperature of the emitting gas. Long-slit spectroscopic data of intermediate resolution are more sensitive and more reliably calibrated to perform temperature estimates and abundance analysis. The combination of echelle and long-slit data provides an opportunity to interpret the abundance results in the light of the internal kinematics of the region while providing important checks on the spectrophotometric calibration.

The development of IFU instruments has provided the spatial coverage required to study extended galactic or extragalactic starforming regions (see e.g. Cairós et al. 2010; García-Benito et al. 2010; Monreal-Ibero et al. 2010, 2011; Pérez-Gallego et al. 2010; Relaño et al. 2010; Rosales-Ortega et al. 2010, 2011; LópezSánchez et al. 2011; Pérez-Montero et al. 2011; Sánchez et al. 2011). However, slit spectroscopy (with medium or high spectral resolution) is a valid option for spectrophotometric analysis. This occurs when the object is very compact, or even extended but with few star-forming knots, and when good spatial and spectral resolution together with a simultaneous wide spectral coverage are required (see e.g. Hägele et al. 2006, 2007, 2008, 2009, 2010, 2011; Cumming et al. 2008; Hägele 2008; López-Sánchez \& Esteban 2009b, 2010a; Pérez-Montero et al. 2009; Firpo et al. 2010; Paper I; López-Sánchez 2010; López-Sánchez \& Esteban 2010b).

In this paper, we perform a detailed analysis of the physical characteristics of the ionized gas in Haro 15, distinguishing among individual components and global line fluxes. Electron temperatures and densities were estimated and used to derive ionic and total abundances for all the different species such as $\mathrm{O}, \mathrm{S}, \mathrm{Ne}$ and $\mathrm{Ar}$. In what follows, we describe the details of the observations and the relevant data reduction procedures. Section 3 explains the different methods used to determine the physical conditions of the ionized gas which lead to the calculation of their chemical abundances, together with their uncertainties. The results for each region from each applied technique are discussed in Section 4, and the summary and conclusions are presented in Section 5.

\section{OBSERVATIONS AND DATA REDUCTION}

We obtained a long-slit moderate resolution spectrum using the wide-field CCD (WFCCD) camera (2005 September 28) mounted on the 100-inch du Pont Telescope, Las Campanas Observatory (LCO). The observations correspond to knots $\mathrm{B}$ and $\mathrm{C}$ of the BCD galaxy Haro 15 (see fig. 1 of Paper I). The TEK5 detector was used covering the wavelength range 3800-9300 $\AA$ (centred at $\lambda_{\mathrm{c}}=6550 \AA$ ). The effective slit width was 1 arcsec giving a spectral resolution of $R \simeq 900(\Delta \lambda=7.5 \AA$ at $\lambda 6700 \AA)$, as measured from the full width at half-maximum (FWHM) of the $\mathrm{He}-\mathrm{Ne}-\mathrm{Ar}$ comparison lines taken for wavelength calibration purposes. Observing conditions were good, with an average seeing of 1 arcsec and photometric sky conditions.

Bias and dome flat-field frames were taken at the beginning of the night. The images were processed and analysed with $\mathrm{IRAF}^{1}$ routines following standard procedures. The procedure includes the removal of cosmic rays, bias subtraction and division by a normalized flatfield and wavelength calibration. To subtract the sky emission we defined two windows on both sides of the 2D spectrum of each observed knot. For knot B the sky subtraction is very good. The final spectrum shows relatively weak residuals of the $\mathrm{OH}$ sky telluric emission lines which do not affect any important emission line from the star-forming region. In the case of knot $\mathrm{C}$, we have a very good sky subtraction for the blue part of the spectrum $(\lambda<7000 \AA)$. We do not measure any emission line in the red part of this spectrum. The goodness of the sky subtractions was taken into account in the estimated errors of the measurements of the emission lines. The standard star EG 131 was observed for flux calibration purposes and the exposure time for this flux standard star was $180 \mathrm{~s}$. The spectra were corrected for atmospheric extinction and flux calibrated.

A certain degree of second-order contamination is present in our long-slit spectrum at wavelengths larger than about $6000 \AA$. We are not able to deconvolve the different contributions of each order in the red end of the spectral range; thus, the measured fluxes of the emission lines in that part of the spectrum may have been slightly overestimated, but these contributions are not very important for the line ratios.

\footnotetext{
${ }^{1}$ Image Reduction and Analysis Facility, distributed by NOAO, operated by AURA, Inc., under agreement with NSF.
} 
Table 1. Journal of observations for the knots. The first column is the observation mode, the second column is the nomenclature used in Paper I, the third column is the observed date, the fourth column is the exposure time for the knots and the fifth column is the air masses.

\begin{tabular}{ccccc}
\hline Mode & Knots & Date & Exposure time & $\sec z$ \\
\hline Long-slit & B,C & 2005 September 28 & $2 \times 1200+1 \times 900$ & 1.5 \\
Echelle & $\mathrm{A}$ & 2006 July 19 & 1800 & 1.2 \\
& $\mathrm{~B}$ & 2006 July 19 & 1800 & 1.1 \\
& $\mathrm{C}$ & 2006 July 19 & 1800 & 1.1 \\
& $\mathrm{E}$ & 2006 July 20 & 1800 & 1.2 \\
\hline
\end{tabular}

Five different regions in Haro 15 (see fig. 1 of Paper I) were observed with high-resolution spectroscopy obtained using an echelle spectrograph attached to the 100-inch du Pont Telescope, LCO, in 2006 July 19 and 20. The spectral range of the observations is 3400-10000 $\AA$. This spectral range guarantees the simultaneous measurement of the nebular emission lines from [O II] $\lambda \lambda 3727,3729$ to $\left[\mathrm{S}_{\mathrm{III}}\right] \lambda \lambda 9069,9532 \AA$ at both ends of the spectrum, in the very same region of the galaxy. Observing conditions were good with an average seeing of 1 arcsec and photometric nights.

A detailed description about the echelle data and the reduction procedure can be found in Paper I. The effective slit width and length were 1 and 4 arcsec, respectively, and the spectral resolution achieved in our du Pont echelle data was $R \simeq 25000(\Delta \lambda=0.25 \AA$ at $\lambda 6000 \AA$ ), as measured from the FWHM of the Th-Ar comparison lines taken for wavelength calibration purposes. The spectra were obtained as single exposures of $1800 \mathrm{~s}$ each. Flux calibration was performed by observing, with an exposure time of $1200 \mathrm{~s}$, the CALSPEC spectrophotometric standard star Feige 110 (Bohlin, Dickinson \& Calzetti 2001). This star flux is tabulated every $2 \AA$, which is ideal for calibrating high-resolution echelle spectra. In addition, Th-Ar comparison spectra, milky flats (sky flats obtained with a diffuser, during the afternoon) and bias frames were taken every night. A journal of observations is shown in Table 1 . The corrected data were reduced with IRAF routines following procedures similar to those described in Firpo, Bosch \& Morrell (2005). It must be noted that data obtained for the fainter knot $\mathrm{F}$ have not sufficient $\mathrm{S} / \mathrm{N}$ as to allow flux measurements with the accuracy needed to perform an analysis of the physical conditions of the gas and have therefore been excluded from this paper.

\section{RESULTS}

\subsection{Line intensities and reddening correction}

The one-dimensional spectra of knots $\mathrm{B}$ and $\mathrm{C}$ extracted from the WFCCD long-slit data are shown in Fig. 1. The spectral plots also include some of the relevant identified emission lines.

For these long-slit data the emission-line fluxes were measured using the splot task in IRAF following the procedure described in Hägele et al. (2006). We used two methods to integrate the emissionline flux: (i) in the case of an isolated line or two blended and unresolved lines, the intensity was calculated integrating between two points given by the position of the local continuum placed by eye; (ii) in the case of two blended and resolved lines, we used a multiple Gaussian fit procedure to estimate individual fluxes. Both procedures are well detailed in Hägele et al. (2006, 2008), hereafter $\mathrm{H} 06$ and H08, respectively). In the case of the echelle data we also followed the procedure described in (i) to measure the flux of a given line including all the different kinematical components. This flux will be referred to as 'global' in this work.

The statistical errors associated with the observed emission fluxes have been calculated using the expression

$\sigma_{1}=\sigma_{\mathrm{c}} N^{1 / 2}[1+E W /(N \Delta)]^{1 / 2}$

following the procedure described in Pérez-Montero \& Díaz (2003) where $\sigma_{1}$ is the error in the observed line flux, $\sigma_{\mathrm{c}}$ represents the standard deviation in a box near the measured emission line and stands for the error in the continuum placement, $N$ is the number of pixels used in the measurement of the line flux, $E W$ is the line equivalent width expressed in $\AA$ and $\Delta$ is the wavelength dispersion in Å per pixel (González-Delgado et al. 1994).

Following the iterative procedure presented in Firpo et al. (2010), we measured the fluxes and estimated the errors associated with each kinematical component identified in the high-resolution echelle data for the strongest emission lines (see Paper I). For those lines with $\mathrm{S} / \mathrm{N}$ too low to perform a self-consistent fitting of the different kinematical components present in their emission profiles, the iterative process used to deconvolve the different components did not yield meaningful results. For these weak emission lines we used the fits found for the strongest emission lines in Paper I with similar ionization degree as the initial guess as input for the task ngaussfit of IRAF. With these we fit the emission profiles of the weak lines fixing the centroids and widths of the corresponding initial approximation, allowing only the profile amplitudes to vary.

We assumed a two-ionization-zone scheme to select the appropriate initial approximation to fit the weak lines: the low-ionization zone where the [O II], [O II], [N II], [S II], [S III] and [Ar III] forbidden lines are originated, and the high-ionization zone where the helium recombination lines and the [O III] and [Ne III] forbidden lines are emitted. We used the self-consistent solution found in Paper I for $\mathrm{H} \alpha,\left[\mathrm{N}_{\mathrm{II}}\right]$ or $\left[\mathrm{S}_{\mathrm{II}}\right]$ (depending on the minimization of the fitting errors) in the low-ionization zone, and [O III] in the high-ionization one.

The upper panels of Figs 2 and 3 show the [O III] $\lambda 5007 \AA$ emission lines in the flux-velocity plane with their kinematical components overlapped for knots A and B, respectively. In the lower panels of these figures, we show examples of the fittings performed for $\mathrm{He}_{\mathrm{I}} \lambda 5876 \AA$ and $\left[\mathrm{O}_{\mathrm{III}}\right] \lambda 4363 \AA$ for knots A and B, respectively, using the approximations shown in the upper panels as the initial approximations. We can appreciate that this procedure for the weak emission lines gives very good fittings with small residuals.

The existence of an underlying stellar population is suggested by the detection of absorption features that depress the Balmer emission lines in the long-slit and echelle spectra. Therefore, the errors introduced by this effect in the measurement of line intensities were minimized by defining a pseudo-continuum at the base of the hydrogen emission lines (see H06). The presence of the wings of the absorption lines implies that, even though we have used a pseudocontinuum, there is still a fraction of the emitted flux that we are not able to measure accurately (see discussion in Díaz 1988). This fraction is not the same for all lines, nor are the ratios between the absorbed fractions and the emission. In H06 it was estimated that the difference in Balmer line fluxes calculated using the defined pseudo-continuum or a multi-Gaussian fit to the absorption and emission components lies within the errors. At any rate, for the Balmer emission lines we have doubled the estimated error, $\sigma_{1}$, as a conservative approach to consider the uncertainties introduced by the presence of the underlying stellar population.

The reddening coefficient $[c(\mathrm{H} \beta)]$ was calculated assuming the galactic extinction law of Miller \& Mathews (1972) with 

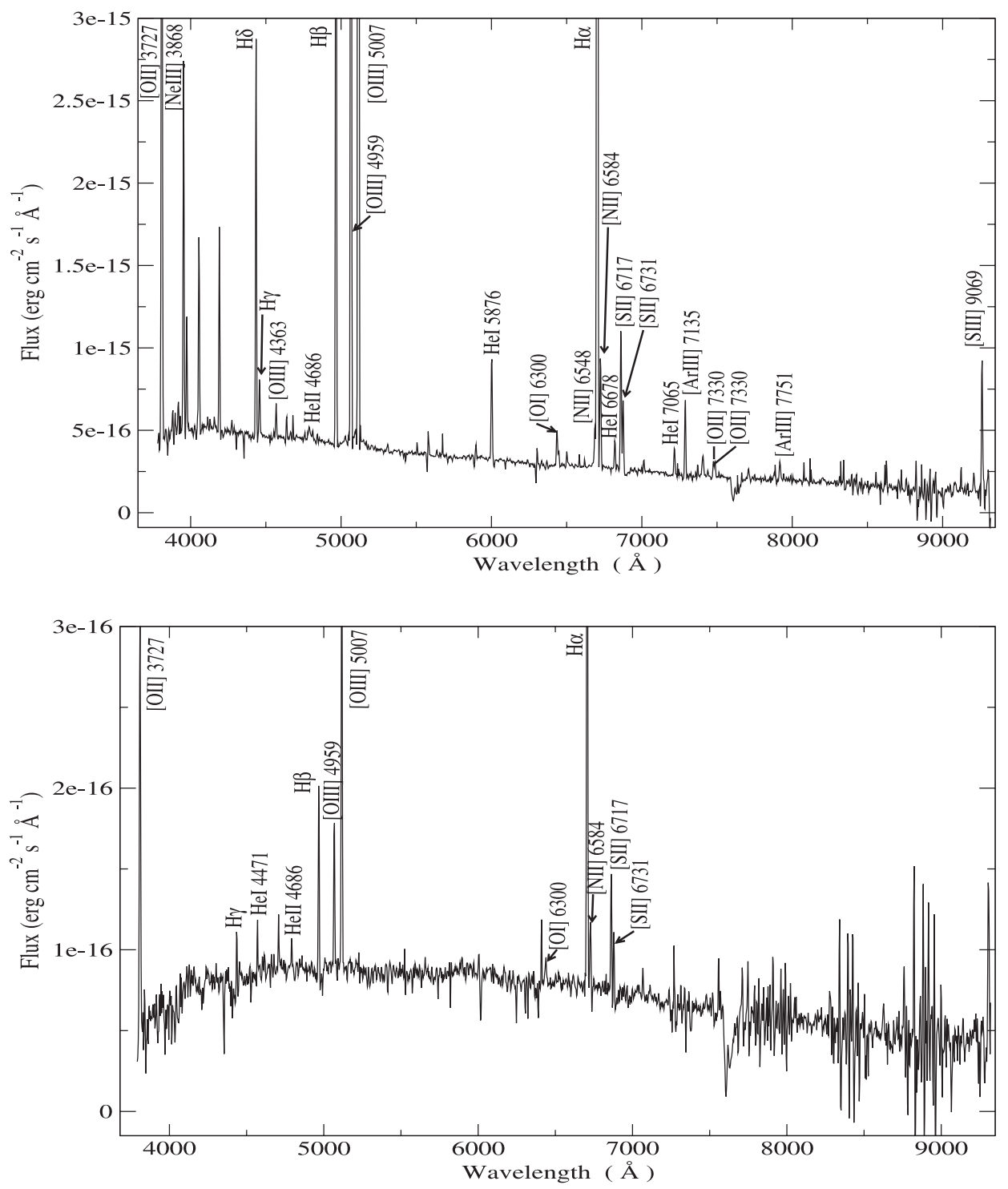

Figure 1. Long-slit spectra of knots B and C of Haro 15 (upper and lower panel, respectively).

$R_{\mathrm{v}}=3.2$. We obtained a value for $c(\mathrm{H} \beta)$ in each case by performing a least-squares fit to the observed ratio between $F\left(\lambda_{B}\right)$ and $F(\mathrm{H} \beta)$, where $B$ denotes the different Balmer lines, to their theoretical values computed by Storey \& Hummer (1995) using an iterative method to estimate $n_{\mathrm{e}}$ and $T_{\mathrm{e}}$ in each case (Hägele 2008). Whenever available, initial $n_{\mathrm{e}}$ and $T_{\mathrm{e}}$ guesses were taken from the measured [S II] $\lambda \lambda 6717,6731$ and [O III] $\lambda \lambda 4363,4959,5007$ line fluxes. We have considered $n_{\mathrm{e}}$ equal to $n([\mathrm{~S}$ II] $)$. Owing to the large error introduced by the presence of the underlying stellar population, only the four strongest Balmer emission lines $(\mathrm{H} \alpha, \mathrm{H} \beta, \mathrm{H} \gamma$ and $\mathrm{H} \delta$ ) have been taken into account.

The emission-line fluxes of the observed knots of Haro 15 are listed in Table 2 for the long-slit data, and in Tables 3-6 for the echelle data. Each table lists the reddening corrected emission-line ratios for each measurement, either for the global or kinematical component, together with the reddening constant and its error taken as the uncertainties of the least-squares fit, and the reddening corrected $\mathrm{H} \beta$ intensity. The first two columns share the same information in Tables 2-6. Column 1 lists the wavelength and identification of the measured lines. The adopted reddening curve, $f(\lambda)$, normalized to $\mathrm{H} \beta$, is given in column 2. The following columns show the equivalent widths $(E W)$ in $\AA$, the reddening corrected emission-line intensities relative to $\mathrm{H} \beta$ and their corresponding errors obtained by propagating in quadrature the observational errors in the emission-line fluxes and the reddening constant uncertainties for different knots/components accordingly. We have not taken into account errors in the theoretical intensities since they are lower than the observational ones.

As the $[\mathrm{S}$ III] $\lambda 9532 \AA$ line is outside the spectral range of the long-slit data, all the physical parameters of knot $\mathrm{B}$ that depend on this emission line were calculated using the theoretical ratio between this line and $\left[\mathrm{S}_{\mathrm{III}}\right] \lambda 9069 \AA, I(9532) \approx 2.44 \times I(9069)$ (Osterbrock 1989). In the case of knot C, we were not able to measure the emission-line flux of $\left[\mathrm{S}_{\text {III }}\right] \lambda 9069 \AA$ with acceptable accuracy. Thus, we do not include this line either in the table or in our calculations. For this knot, it was impossible to measure the $\left[\mathrm{N}_{\mathrm{II}}\right] \lambda 6548 \AA$ emission line since it is blended with $\mathrm{H} \alpha$. We corrected the $\mathrm{H} \alpha$ flux for the contamination by this nitrogen line estimating this contribution from $\left[\mathrm{N}_{\mathrm{II}}\right] \lambda 6584 \AA$ using the theoretical ratio, $I(6584) \approx 2.9 \cdot I(6548)$ (Osterbrock 1989$)$. 

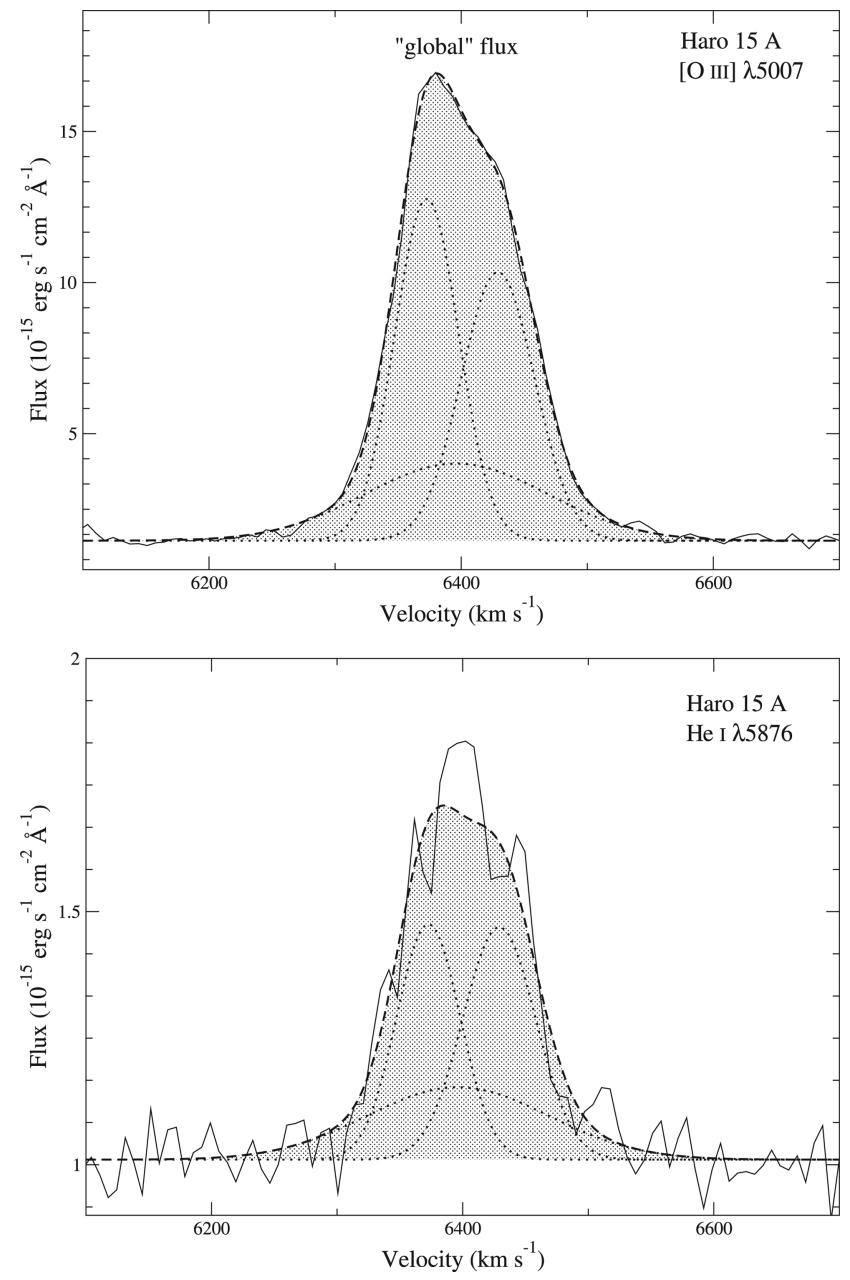

Figure 2. [O III] $\lambda 5007 \AA$ (upper panel) and He I $\lambda 5876 \AA$ (lower panel) emission lines of knot $\mathrm{A}$ in the flux-velocity plane with their kinematical decomposition and the sum (hatched area) of the different components superposed.

We can appreciate in the echelle data that the $\left[\mathrm{S}_{\mathrm{II}}\right] \lambda 6731$ A emission line is affected by $\mathrm{OH}$ sky telluric emission lines present in the same spectral range due to the galaxy redshift. We used the standard star Feige 110 (sdOB) as telluric star since this star has no stellar lines in this spectral range. Using IRAF telluric routing, we corrected the echelle spectra by this effect. The following step was to resort to the ngaussfit task using the fitting solution for $\left[\mathrm{S}_{\mathrm{II}}\right] \lambda 6717 \AA$ as the initial approximation to fit the $\left[\mathrm{S}_{\mathrm{II}}\right] \lambda 6731 \AA$ line profile fixing the centroids and widths of the components, allowing only the amplitudes to be fitted. The emission lines with relative flux errors higher than 45 per cent were not taken into account for the calculations. The exception is the $\left[\mathrm{O}_{\text {IIII }}\right] \lambda 4363 \AA$ auroral emission line in knot $\mathrm{B}$, which, albeit faint, is essential to estimate the $[\mathrm{O}$ III] line temperature. We were not able to measure the $\left[\mathrm{O}_{\text {II }}\right] \lambda \lambda 7319,7330 \AA$ auroral emission lines for any echelle spectra. In knots $\mathrm{C}$ and $\mathrm{E}$, the $[\mathrm{S} \mathrm{II}] \lambda \lambda 9069,9532 \AA$ lines were not detected due to the low $\mathrm{S} / \mathrm{N}$ of these spectra in this spectral range. In these knots the $\left[\mathrm{O}_{\mathrm{II}}\right] \lambda 4959 \AA$ $\mathrm{\AA}$ emission-line fluxes were calculated from the $[\mathrm{O}$ III] $\lambda 5007 \AA$ line using the theoretical ratio, $I(5007) \approx 3 \times I(4959)$ (Osterbrock 1989). In knot A, the weak $\mathrm{H} \delta$ emission line was not considered for calculations. For knot E, the narrow 2 component and the global measure present $c(\mathrm{H} \beta)$ values slightly lower than zero but still compatible with this value
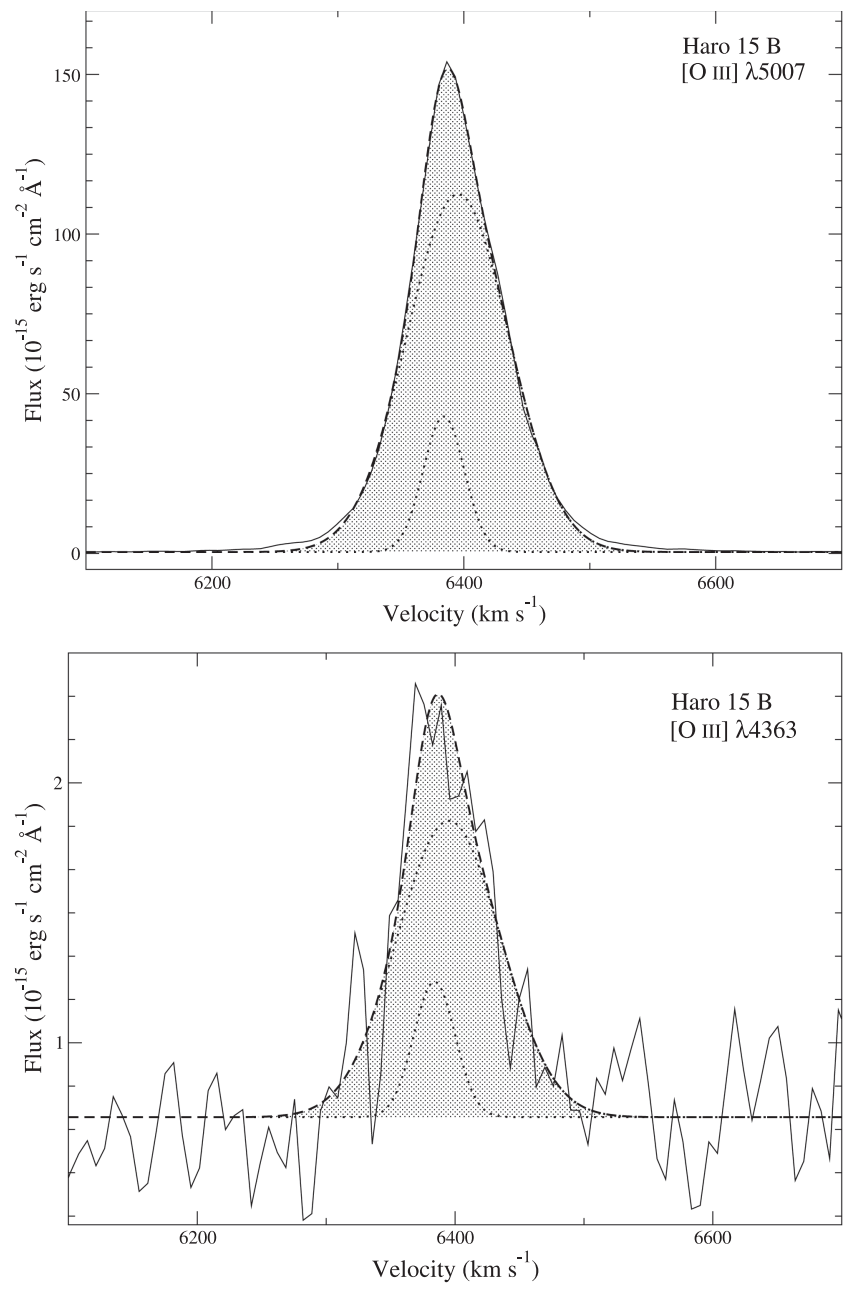

Figure 3. $[\mathrm{O} \mathrm{III}] \lambda 5007 \AA$ (upper panel) and $[\mathrm{O} \mathrm{III}] \lambda 4363 \AA$ (lower panel) emission lines of knot B in the flux-velocity plane with their kinematical decomposition and the sum (hatched area) of the different components superposed.

within the observational errors. In this case we considered $c(\mathrm{H} \beta)$ as zero.

\subsection{Physical conditions of the ionized gas}

We studied the physical conditions of the emitting medium in four star-forming knots of Haro 15 using long-slit (only for knots B and C) and echelle data. With the echelle data, we analysed the conditions in each knot from the global and kinematical decomposition measurements following the results derived in Paper I. In what follows we will explain the different methods used to estimate the electron temperatures $\left(T_{\mathrm{e}}\right)$ and densities $\left[N_{\mathrm{e}} \approx n\left(\left[\mathrm{~S}_{\mathrm{II}}\right]\right)\right]$ (see discussion in Firpo 2011, and references therein).

(i) When the weak auroral lines needed to implement the direct method could be measured we derived the physical conditions using the direct method as described in H08 and Hägele et al. (2011, hereafter H11). This procedure is based on the five-level statistical equilibrium atom approximation in the task temden of the software package IRAF (De Robertis, Dufour \& Hunt 1987; Shaw \& Dufour 1995). We took the uncertainties associated with the measurement of the emission-line fluxes and the reddening correction 
Table 2. Relative reddening corrected line intensities $[F(\mathrm{H} \beta)=I(\mathrm{H} \beta)=10000]$ for the global measure of the long-slit spectrum of knots B and C.

\begin{tabular}{|c|c|c|c|c|c|c|c|}
\hline \multirow{2}{*}{$\lambda(\AA)$} & \multirow[b]{2}{*}{$f(\lambda)$} & \multicolumn{3}{|c|}{ B } & \multicolumn{3}{|c|}{$\mathrm{C}$} \\
\hline & & $\begin{array}{l}-E W \\
(\AA)\end{array}$ & $I(\lambda)$ & $\begin{array}{c}\text { Error } \\
\text { (per cent) }\end{array}$ & $\begin{array}{l}-E W \\
(\AA)\end{array}$ & $I(\lambda)$ & $\begin{array}{c}\text { Error } \\
\text { (per cent) }\end{array}$ \\
\hline $3727\left[\mathrm{O}_{\text {II }}{ }^{a}\right.$ & 0.271 & 109.1 & $11940 \pm 120$ & 1.0 & 45.3 & $25400 \pm 2100$ & 8.3 \\
\hline 3835 H9 & 0.246 & 3.8 & $470 \pm 20$ & 5.0 & - & - & - \\
\hline 3868 [Ne III] & 0.238 & 34.0 & $4490 \pm 10$ & 0.2 & - & - & - \\
\hline $3889 \mathrm{He} \mathrm{I}+\mathrm{H} 8$ & 0.233 & 9.4 & $1260 \pm 70$ & 5.1 & - & - & - \\
\hline $3970[\mathrm{Ne}$ III] $]+\mathrm{H} \epsilon$ & 0.215 & 21.1 & $2535 \pm 4$ & 0.2 & - & - & - \\
\hline $4068\left[\mathrm{~S}_{\mathrm{II}}\right]$ & 0.195 & 0.7 & $90 \pm 20$ & 19.6 & - & - & - \\
\hline $4102 \mathrm{H} \delta$ & 0.188 & 21.1 & $2400 \pm 30$ & 1.3 & - & - & - \\
\hline $4340 \mathrm{H} \gamma$ & 0.142 & 41.5 & $4480 \pm 20$ & 0.4 & 6.0 & $3850 \pm 660$ & 17.1 \\
\hline 4363 [O III] & 0.138 & 6.3 & $690 \pm 40$ & 6.0 & - & - & - \\
\hline $4471 \mathrm{He} \mathrm{I}$ & 0.106 & 2.8 & $310 \pm 20$ & 5.2 & 3.8 & $2840 \pm 570$ & 19.9 \\
\hline $4658[\mathrm{Fe}$ III] & 0.053 & 0.3 & $36 \pm 6$ & 17.8 & - & - & - \\
\hline $4686 \mathrm{He}_{\text {II }}$ & 0.045 & 1.5 & $150 \pm 20$ & 12.1 & 1.6 & $1260 \pm 330$ & 26.1 \\
\hline $4713\left[\mathrm{Ar}\right.$ IV] $+\mathrm{He} \mathrm{I}_{\mathrm{I}}$ & 0.038 & 0.6 & $64 \pm 7$ & 11.4 & - & - & - \\
\hline 4740 [Ar IV] & 0.031 & 0.9 & $90 \pm 10$ & 14.1 & - & - & - \\
\hline $4861 \mathrm{H} \beta$ & 0.000 & 114.1 & $10000 \pm 30$ & 0.3 & 14.6 & $10000 \pm 540$ & 5.4 \\
\hline 4959 [O III] & -0.024 & 221.3 & $21220 \pm 80$ & 0.4 & 8.3 & $6680 \pm 210$ & 3.1 \\
\hline 5007 [O III] & -0.035 & 539.1 & $51340 \pm 90$ & 0.2 & 27.9 & $22040 \pm 470$ & 2.1 \\
\hline $5876 \mathrm{He}_{\mathrm{I}}$ & -0.209 & 22.5 & $1240 \pm 30$ & 2.3 & - & - & - \\
\hline $6300[\mathrm{OI}]$ & -0.276 & 6.8 & $370 \pm 20$ & 4.3 & 2.5 & $1540 \pm 300$ & 19.1 \\
\hline 6312 [S III] & -0.278 & 2.3 & $120 \pm 20$ & 15.5 & - & - & - \\
\hline 6364 [O I] & -0.285 & 2.2 & $120 \pm 10$ & 9.6 & - & - & - \\
\hline 6548 [N II] & -0.311 & 8.3 & $420 \pm 20$ & 4.8 & - & - & - \\
\hline $6563 \mathrm{H} \alpha$ & -0.313 & 565.8 & $27620 \pm 40$ & 0.1 & 52.6 & $28500 \pm 820$ & 2.9 \\
\hline 6584 [N II] & -0.316 & 23.4 & $1180 \pm 20$ & 1.8 & 5.8 & $3230 \pm 370$ & 11.5 \\
\hline $6678 \mathrm{He} \mathrm{I}$ & -0.329 & 5.5 & $260 \pm 10$ & 4.7 & - & - & - \\
\hline 6717 [S II] & -0.334 & 32.1 & $1450 \pm 20$ & 1.7 & 10.2 & $5210 \pm 610$ & 11.8 \\
\hline 6731 [S II] & -0.336 & 13.7 & $620 \pm 50$ & 8.0 & 5.1 & $2610 \pm 490$ & 18.9 \\
\hline 7065 He I & -0.377 & 6.3 & $250 \pm 20$ & 7.2 & - & - & - \\
\hline 7136 [Ar III] & -0.385 & 19.4 & $710 \pm 20$ & 3.0 & - & - & - \\
\hline $7281 \mathrm{He} \mathrm{I}^{b}$ & -0.402 & 1.1 & $42 \pm 5$ & 11.9 & - & - & - \\
\hline $7319\left[\right.$ [O II] $^{c}$ & -0.406 & 4.0 & $148 \pm 6$ & 3.9 & - & - & - \\
\hline $7330[\mathrm{O} \mathrm{II}]^{d}$ & -0.407 & 3.1 & $116 \pm 4$ & 3.2 & - & - & - \\
\hline 7751 [Ar III] & -0.451 & 5.8 & $190 \pm 10$ & 7.0 & - & - & - \\
\hline 9069 [S III] & -0.561 & 69.9 & $1200 \pm 120$ & 9.8 & - & - & - \\
\hline \multirow{2}{*}{\multicolumn{2}{|c|}{$\begin{array}{l}I(\mathrm{H} \beta)\left(\mathrm{erg} \mathrm{s}^{-1} \mathrm{~cm}^{-2}\right) \\
c(\mathrm{H} \beta)\end{array}$}} & \multirow{2}{*}{\multicolumn{3}{|c|}{$\begin{array}{c}4.49 \times 10^{-14} \\
0.29 \pm 0.01\end{array}$}} & \multicolumn{3}{|c|}{$0.11 \times 10^{-14}$} \\
\hline & & & & & & $0.24 \pm 0.12$ & \\
\hline
\end{tabular}

${ }^{a}\left[\mathrm{O}_{\text {II }}\right] \lambda \lambda 3726+3729 ;{ }^{b}$ possibly blend with an unknown line

${ }^{c}\left[\mathrm{O}_{\mathrm{II}}\right] \lambda \lambda 7318+7320 ;{ }^{d}[\mathrm{O}$ II $] \lambda \lambda 7330+7331$.

as error sources, and we propagated them through our calculations. The adopted collisional atomic coefficients are the same as used in $\mathrm{H} 08$.

(ii) When some of the weak auroral lines were not detected in the spectrum of an observed region, and the line temperatures could not be computed using the direct method, we used relations between temperatures derived using photoionization models (Pérez-Montero \& Díaz 2003, 2005), or empirical and semi-empirical relations (Hägele et al. 2006; Díaz et al. 2007; Pilyugin 2007). Pérez-Montero \& Díaz $(2003,2005)$ examined some relationships between temperatures used for ionic abundance determinations, adapting these relations to the latest photoionization model results at that moment and using the most recent atomic coefficients. We have to stress that the errors of the line temperatures derived using these modeldependent relations are formal errors calculated from the measured line intensity errors applying error propagation formulae, without assigning any error to the temperature calibration itself. We estimated line temperatures following the method described in H06 and H08 which takes advantage of the temperatures derived us- ing the direct method in combination with either model-based or empirical relations.

(iii) When no auroral emission line was detected in the spectrum, we used empirical methods to estimate the electron temperatures:

(a) If the strong emission lines of $\left[\mathrm{S}_{\mathrm{II}}\right],[\mathrm{S} \mathrm{III}],\left[\mathrm{O}_{\mathrm{II}}\right]$ and $\left[\mathrm{O}_{\mathrm{III}}\right]$ are available in the spectrum of the studied region, we can use the relation between the empirical parameter $\mathrm{SO}_{23}\left(=\mathrm{S}_{23} / \mathrm{O}_{23}\right.$; defined by Díaz \& Pérez-Montero 2000) and the [S III] line temperature developed by Díaz et al. (2007, hereafter P07):

$t_{\mathrm{e}}\left(\left[\mathrm{S}_{\mathrm{III}}\right]\right)=0.596-0.283 \log \mathrm{SO}_{23}+0.199\left(\log \mathrm{SO}_{23}\right)^{2}$.

This relationship was derived for high-metallicity regions. However, from fig. 9 of D07, we can appreciate that this relation is also valid for the $\mathrm{H}$ II galaxies in the upper end of the $\mathrm{SO}_{23}$ distribution for this kind of objects (where their dispersion is the lowest) and whose derived values of $\mathrm{SO}_{23}$ are in the validity range of the relation. Fortunately this is the case for the $\mathrm{SO}_{23}$ empirical parameter as measured in the star-forming knots of Haro 15. 


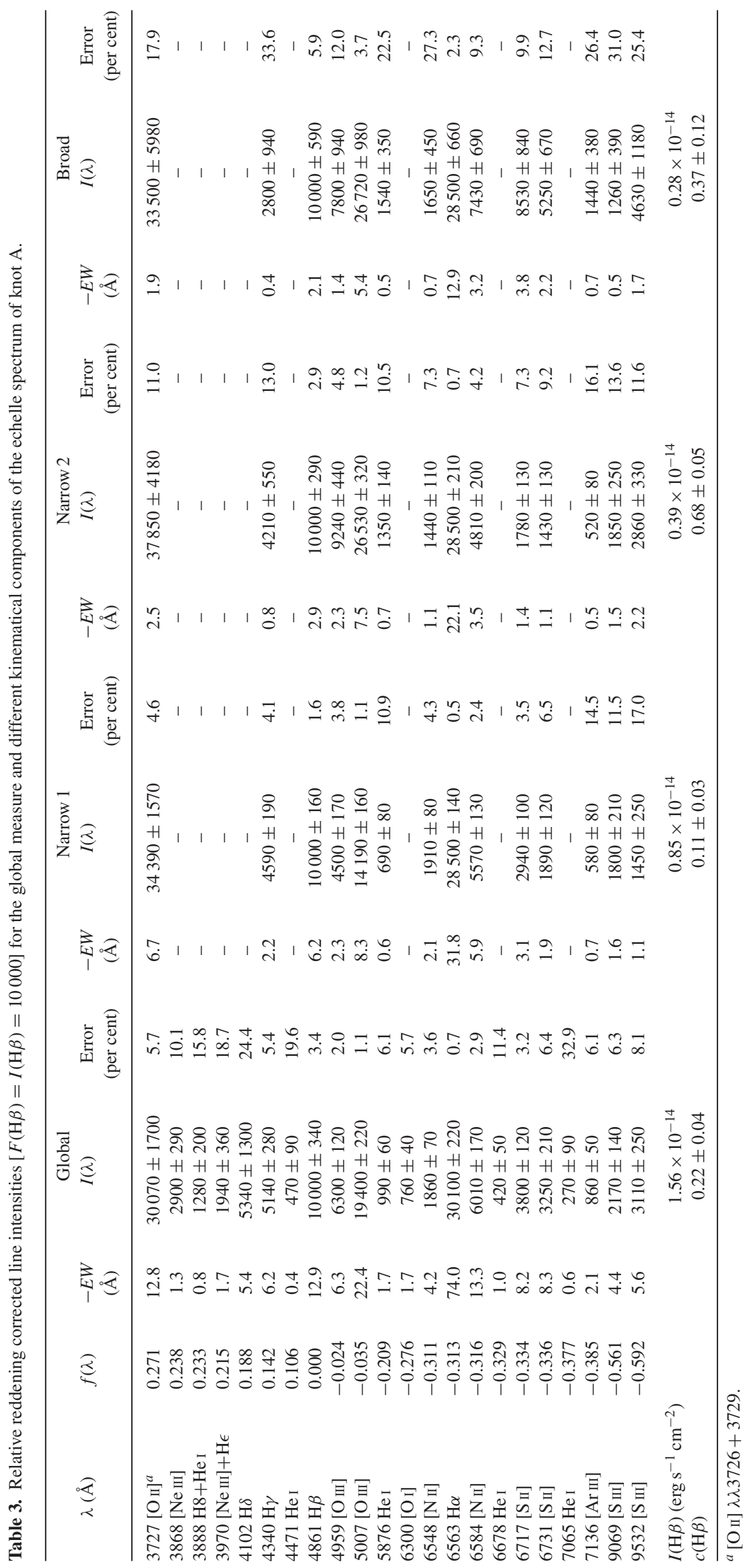


Table 4. Relative reddening corrected line intensities $[F(H \beta)=I(H \beta)=10000]$ for the global measure and different kinematical components of the echelle spectrum of knot B.

\begin{tabular}{|c|c|c|c|c|c|c|c|c|c|c|}
\hline$\lambda(\AA)$ & $f(\lambda)$ & $\begin{array}{c}-E W \\
(\AA)\end{array}$ & $\begin{array}{l}\text { Global } \\
I(\lambda)\end{array}$ & $\begin{array}{c}\text { Error } \\
\text { (per cent) }\end{array}$ & $\begin{array}{c}-E W \\
(\AA)\end{array}$ & $\begin{array}{l}\text { Narrow } \\
I(\lambda)\end{array}$ & $\begin{array}{c}\text { Error } \\
\text { (per cent) }\end{array}$ & $\begin{array}{c}-E W \\
(\AA)\end{array}$ & $\begin{array}{l}\text { Broad } \\
I(\lambda)\end{array}$ & $\begin{array}{c}\text { Error } \\
\text { (per cent) }\end{array}$ \\
\hline 3835 H9 & 0.246 & 1.0 & $360 \pm 20$ & 4.4 & - & - & - & - & - & - \\
\hline 3868 [Ne III] & 0.238 & 12.4 & $5590 \pm 180$ & 3.3 & 1.6 & $4210 \pm 650$ & 15.4 & 8.4 & $5850 \pm 270$ & 4.6 \\
\hline $3889 \mathrm{He}_{\mathrm{I}}+\mathrm{H} 8$ & 0.233 & 5.1 & $1570 \pm 40$ & 2.6 & - & - & - & - & - & - \\
\hline $4102 \mathrm{H} \delta$ & 0.188 & 9.7 & $2770 \pm 120$ & 4.3 & 1.4 & $2220 \pm 340$ & 15.2 & 5.7 & $2530 \pm 110$ & 4.5 \\
\hline $4340 \mathrm{H} \gamma$ & 0.142 & 37.8 & $4830 \pm 120$ & 2.5 & 5.6 & $5310 \pm 160$ & 3.0 & 16.7 & $4430 \pm 60$ & 1.3 \\
\hline 4363 [O III] & 0.138 & 4.4 & $780 \pm 130$ & 16.3 & 0.4 & $470 \pm 210$ & 45.8 & 2.4 & $720 \pm 90$ & 12.5 \\
\hline $4471 \mathrm{He}_{\mathrm{I}}$ & 0.106 & 3.0 & $470 \pm 20$ & 3.6 & - & - & - & - & - & - \\
\hline $4713[\mathrm{Ar} \mathrm{IV}]+\mathrm{He}_{\mathrm{I}}$ & 0.038 & 2.6 & $340 \pm 30$ & 8.4 & - & - & - & - & - & - \\
\hline $4861 \mathrm{H} \beta$ & 0.000 & 81.3 & $10000 \pm 100$ & 1.0 & 14.1 & $10000 \pm 160$ & 1.6 & 45.6 & $10000 \pm 70$ & 0.7 \\
\hline $6312[\mathrm{~S} \mathrm{III]}$ & -0.278 & 1.8 & $130 \pm 30$ & 20.2 & 0.7 & $200 \pm 40$ & 22.2 & 1.0 & $100 \pm 30$ & 24.1 \\
\hline $6548\left[\mathrm{~N}_{\text {II }}\right]$ & -0.311 & 2.4 & $185 \pm 5$ & 2.6 & 1.6 & $420 \pm 20$ & 5.7 & 1.6 & $160 \pm 20$ & 13.0 \\
\hline $6563 \mathrm{H} \alpha$ & -0.313 & 358.0 & $28620 \pm 40$ & 0.1 & 73.5 & $29350 \pm 270$ & 0.9 & 184.8 & $28010 \pm 130$ & 0.5 \\
\hline 6584 [N II] & -0.316 & 7.7 & $620 \pm 10$ & 1.9 & 6.0 & $1570 \pm 50$ & 3.4 & 4.0 & $400 \pm 30$ & 6.2 \\
\hline $6678 \mathrm{He}_{\mathrm{I}}$ & -0.329 & 4.8 & $320 \pm 3$ & 1.1 & 0.5 & $130 \pm 30$ & 20.7 & 4.6 & $430 \pm 20$ & 3.8 \\
\hline 6717 [S II] & -0.334 & 10.6 & $730 \pm 10$ & 1.3 & 7.4 & $1920 \pm 50$ & 2.4 & 3.6 & $360 \pm 30$ & 8.1 \\
\hline 6731 [S II] & -0.336 & 8.4 & $590 \pm 20$ & 3.8 & 5.2 & $1410 \pm 50$ & 3.8 & 2.6 & $270 \pm 30$ & 12.6 \\
\hline $7065 \mathrm{He}_{\mathrm{I}}$ & -0.377 & 4.9 & $290 \pm 6$ & 1.9 & - & - & - & - & - & - \\
\hline 7136 [Ar III] & -0.385 & 11.0 & $710 \pm 9$ & 1.3 & 4.0 & $980 \pm 50$ & 5.3 & 6.9 & $670 \pm 20$ & 3.4 \\
\hline 7751 [Ar III] & -0.451 & 3.2 & $200 \pm 10$ & 6.4 & - & - & - & - & - & - \\
\hline $9069[\mathrm{~S} \mathrm{III}]$ & -0.561 & 13.9 & $1190 \pm 110$ & 9.3 & 3.1 & $1010 \pm 140$ & 13.5 & 9.2 & $1330 \pm 90$ & 6.8 \\
\hline 9532 [S III] & -0.592 & 21.3 & $2510 \pm 140$ & 5.6 & 6.9 & $2890 \pm 190$ & 6.4 & 13.1 & $2490 \pm 130$ & 5.0 \\
\hline
\end{tabular}

$a_{[\text {II }]} \lambda \lambda 3726+3729$.

Table 5. Relative reddening corrected line intensities $[F(H \beta)=I(H \beta)=10000]$ for the global measure and different kinematical components of the echelle spectrum of knot C.

\begin{tabular}{|c|c|c|c|c|c|c|c|c|c|c|}
\hline \multirow[b]{2}{*}{$\lambda(\AA)$} & \multirow[b]{2}{*}{$f(\lambda)$} & \multicolumn{3}{|c|}{ Global } & \multicolumn{3}{|c|}{ Narrow } & \multicolumn{3}{|c|}{ Broad } \\
\hline & & $\begin{array}{l}-E W \\
(\AA)\end{array}$ & $I(\lambda)$ & $\begin{array}{c}\text { Error } \\
\text { (per cent) }\end{array}$ & $\begin{array}{l}-E W \\
(\AA)\end{array}$ & $I(\lambda)$ & $\begin{array}{c}\text { Error } \\
\text { (per cent) }\end{array}$ & $\begin{array}{c}-E W \\
(\AA)\end{array}$ & $I(\lambda)$ & $\begin{array}{c}\text { Error } \\
\text { (per cent) }\end{array}$ \\
\hline 4959 [O III] & -0.024 & 5.6 & $6880 \pm 390$ & 5.7 & 3.4 & $9820 \pm 510$ & 5.2 & 2.0 & $5250 \pm 610$ & 11.7 \\
\hline 5007 [O III] & -0.035 & 5.6 & $20470 \pm 1270$ & 6.2 & 3.4 & $29370 \pm 1580$ & 5.4 & 2.0 & $15580 \pm 1860$ & 11.9 \\
\hline $6563 \mathrm{H} \alpha$ & -0.313 & 18.1 & $28500 \pm 1150$ & 4.0 & 6.5 & $28500 \pm 710$ & 2.5 & 8.0 & $28500 \pm 800$ & 2.8 \\
\hline $6731\left[\mathrm{~S}_{\text {II }}\right]$ & -0.336 & 1.8 & $2890 \pm 1070$ & 37.0 & 0.7 & $3190 \pm 1300$ & 40.6 & 1.0 & $3890 \pm 1610$ & 41.3 \\
\hline \multirow{2}{*}{\multicolumn{2}{|c|}{$\begin{array}{l}I(\mathrm{H} \beta)\left(\mathrm{erg} \mathrm{s}^{-1} \mathrm{~cm}^{-2}\right) \\
c(\mathrm{H} \beta)\end{array}$}} & & $0.08 \times 10^{-14}$ & & & $0.04 \times 10^{-14}$ & & & $0.04 \times 10^{-14}$ & \\
\hline & & & $0.21 \pm 0.40$ & & & $0.14 \pm 0.25$ & & & $0.38 \pm 0.41$ & \\
\hline
\end{tabular}

We used this $T_{\mathrm{e}}([\mathrm{S} \mathrm{III}])$ to estimate $T_{\mathrm{e}}([\mathrm{O} \mathrm{III}])$ using the relation between these two line temperatures derived by H06:

$t_{\mathrm{e}}([\mathrm{S} I I I])=(1.19 \pm 0.08) t_{\mathrm{e}}([\mathrm{O} I I I])-(0.32 \pm 0.10)$.

The temperature errors have been calculated from the measured line intensity errors, taking also into account the temperature calibration errors.

(b) If the [S III] emission lines are not detected with enough quality, we can use the relation between the $[\mathrm{N}$ II] nebular-to-auroral line intensities as a function of the intensities of the strong nebular oxygen lines for the determination of the [N $\mathrm{NI}$ electron temperature $\left(t_{2}\right)$ applying the empirical relation by Pilyugin (2007, hereafter P07):

$t_{2}=\frac{1.111}{\log Q_{\mathrm{N} I I}-0.892-0.144 \cdot \log t_{2}+0.023 \cdot t_{2}}$,

where $Q_{\mathrm{NII}}$ is the approximation to the $[\mathrm{N}$ II] line intensities ratio $\left(=\left[\mathrm{N}_{\mathrm{II}}\right] \lambda \lambda 6548,84 /\left[\mathrm{N}_{\mathrm{II}}\right] \lambda 5755\right) . Q_{\mathrm{NII}}$ depends on the strong $\left[\mathrm{O}_{\mathrm{II}}\right] \lambda \lambda 3727,29 \AA$ and $[\mathrm{O}$ III] $\lambda \lambda 4959,5007 \AA$ emission lines. 
Table 6. Relative reddening corrected line intensities $[F(H \beta)=I(H \beta)=10000]$ for the global measure and different kinematical components of the echelle spectrum of knot E.

\begin{tabular}{|c|c|c|c|c|c|c|c|c|c|c|}
\hline$\lambda(\AA)$ & $f(\lambda)$ & $\begin{array}{l}-E W \\
(\AA)\end{array}$ & $\begin{array}{l}\text { Global } \\
I(\lambda)\end{array}$ & $\begin{array}{c}\text { Error } \\
\text { (per cent) }\end{array}$ & $\begin{array}{l}-E W \\
(\AA)\end{array}$ & $\begin{array}{l}\text { Narrow } 1 \\
I(\lambda)\end{array}$ & $\begin{array}{c}\text { Error } \\
\text { (per cent) }\end{array}$ & $\begin{array}{c}-E W \\
(\AA)\end{array}$ & $\begin{array}{l}\text { Narrow } 2 \\
I(\lambda)\end{array}$ & $\begin{array}{c}\text { Error } \\
\text { (per cent) }\end{array}$ \\
\hline $4861 \mathrm{H} \beta$ & 0.000 & 7.5 & $10000 \pm 1530$ & 15.3 & 1.8 & $10000 \pm 1270$ & 12.7 & 1.9 & $10000 \pm 1800$ & 18.2 \\
\hline 4959 [O III] & -0.024 & 2.9 & $5750 \pm 730$ & 12.7 & 2.2 & $3440 \pm 350$ & 10.2 & 4.4 & $6900 \pm 380$ & 5.6 \\
\hline 5007 [O III] & -0.035 & 6.9 & $12160 \pm 920$ & 7.6 & 2.2 & $10160 \pm 1050$ & 10.3 & 4.4 & $20670 \pm 1210$ & 5.9 \\
\hline 6548 [N II] & -0.311 & 1.4 & $1420 \pm 470$ & 33.0 & - & - & - & - & - & - \\
\hline $6563 \mathrm{H} \alpha$ & -0.313 & 32.0 & $28500 \pm 1090$ & 3.8 & 15.9 & $28500 \pm 640$ & 2.2 & 11.0 & $28500 \pm 960$ & 3.4 \\
\hline 6584 [N II] & -0.316 & 5.8 & $5270 \pm 1160$ & 22.0 & 1.7 & $2810 \pm 620$ & 22.0 & 3.7 & $9030 \pm 2220$ & 24.6 \\
\hline 6717 [S II] & -0.334 & 6.0 & $6210 \pm 1440$ & 23.2 & 1.4 & $2510 \pm 510$ & 20.4 & 4.3 & $11230 \pm 2880$ & 25.6 \\
\hline $6731[\mathrm{~S}$ II] & -0.336 & 5.2 & $4450 \pm 1400$ & 31.5 & 0.8 & $1880 \pm 790$ & 42.1 & 1.8 & $6640 \pm 2630$ & 39.6 \\
\hline \multicolumn{2}{|c|}{$\begin{array}{l}I(\mathrm{H} \beta)\left(\mathrm{erg} \mathrm{s}^{-1} \mathrm{~cm}^{-2}\right) \\
c(\mathrm{H} \beta)\end{array}$} & & $\begin{array}{c}0.18 \times 10^{-14} \\
0.00\end{array}$ & & & $\begin{array}{c}0.07 \times 10^{-14} \\
0.59 \pm 0.22\end{array}$ & & & $\begin{array}{c}0.07 \times 10^{-14} \\
0.00\end{array}$ & \\
\hline
\end{tabular}

$\left.a_{[\mathrm{O}} \mathrm{II}\right] \lambda 3726+3729$.

As the calibration of Pilyugin's method was derived using data on high-metallicity regions and its validity could be questioned in the low-metallicity regime, we confirmed it in those regions where both methods could be applied, finding good agreement. It is therefore possible to estimate the line temperature of [N II], which apparently characterizes the low-ionization area of the nebula, using Pilyugin's method. D07 found a systematic difference of about $500 \mathrm{~K}$ between the $[\mathrm{N}$ II] line temperature using Pilyugin's method and [S III] temperatures derived using their empirical relation. This difference was accounted for when these determinations were present in our work.

(iv) When we could not measure either any auroral emission line, or any intense lines to derive the electron temperatures from empirical methods, for example in the two weakest star-forming knots, we considered $T_{\mathrm{e}}([\mathrm{O} \mathrm{III}])$ equal to $10^{4} \mathrm{~K}$, since it is a typical value for this kind of objects. Theoretical and empirical relations were therefore used to derive the other line temperatures from the adopted value for $T_{\mathrm{e}}([\mathrm{O} \mathrm{III}])$.

\subsection{Chemical abundance derivation}

To study the global and kinematical component abundances in each knot, we derived the ionic chemical abundances of different species. We used the strongest available emission lines detected in the analysed long-slit and echelle spectra and the task ionic of the STSDAS package in IRAF, based on the five-level statistical equilibrium atom approximation, as described in $\mathrm{H} 08$.

The total abundances were derived taking into account, when required, the unseen ionization stages of each element, resorting to the most widely accepted ionization correction factors (ICFs) for each species, $\mathrm{X} / \mathrm{H}=\mathrm{ICF}\left(\mathrm{X}^{+i}\right) \mathrm{X}^{+i} / \mathrm{H}^{+}$(see Pérez-Montero et al. 2007). The procedure is detailed in the following subsections.

\subsubsection{Helium}

The helium recombination lines arise mainly from pure recombination, although they could have some contribution from collisional excitation and be affected by self-absorption (see Olive \& Skillman 2001, 2004, for a complete treatment of these effects). The electron temperature $T_{\mathrm{e}}([\mathrm{O} \mathrm{III}])$ is taken as representative of the zone where the He emission arises since ratios of recombination lines are only weakly sensitive to electron temperature. We used the equations given by Olive and Skillman to derive the $\mathrm{He}^{+} / \mathrm{H}^{+}$value, using the theoretical emissivities scaled to $\mathrm{H} \beta$ from Benjamin, Skillman $\&$ Smits (1999) and the expressions for the collisional correction factors from Kingdon \& Ferland (1995). To calculate the abundance of twice ionized helium we used equation (9) from Kunth \& Sargent (1983). A summary of the equations used to calculate these ionic abundances is given in appendix B of García-Benito (2009).

It is possible to calculate the abundances of once and twice ionized helium using the $\mathrm{He}_{\mathrm{I}} \lambda \lambda 4471,5876,6678,7065 \AA$ and He II $\lambda 4686 \AA$ emission lines, respectively. When these lines were available, we used them to derive the corresponding ionic abundance. As the observed objects have low densities, and three of the used helium lines have a small dependence with optical depth effects, we did not made any corrections for the fluorescence. We did not correct either for the presence of an underlying stellar population. The total abundance of He was found by adding directly the two ionic abundances, $\mathrm{He} / \mathrm{H}=\left(\mathrm{He}^{+}+\mathrm{He}^{2+}\right) / \mathrm{H}^{+}$. The results obtained for each line and the total $\mathrm{He}$ abundances, along with their corresponding errors, are presented in Table 7 and 8 for the long-slit and echelle data, respectively. These tables also include the adopted value for $\mathrm{He}^{+} / \mathrm{H}^{+}$as the average value, weighted by the errors, of the abundances derived from each $\mathrm{He}$ I emission line.

\subsubsection{Ionic and total chemical abundances from forbidden lines}

We calculated the ionic and total abundances of $\mathrm{O}, \mathrm{S}, \mathrm{N}, \mathrm{Ne}$ and Ar using the estimated line temperatures as described in H08. The Fe ionic abundance was derived using equations (11) and (12) from Izotov, Thuan \& Lipovetsky (1994). In those cases where there are no measurements of the $\left[\mathrm{S}_{\mathrm{II}}\right] \lambda 4068 \AA$ and $\left[\mathrm{O}_{\text {II }}\right] \lambda 7325 \AA$ auroral emission lines, and under the assumption of a homogeneous electron temperature in the low-ionization zone, we took the approximation $T_{\mathrm{e}}([\mathrm{S} \mathrm{II}]) \approx T_{\mathrm{e}}([\mathrm{O}$ II] $)$ (Pérez-Montero \& Díaz 2003), where the [O II] temperature was derived from the $[\mathrm{O} \mathrm{III}]$ ones using the relations given by photoionization models (Pérez-Montero \& Díaz 2003). 
Table 7. Physical properties, and ionic and total chemical abundances derived from forbidden lines and the helium recombination lines for the global measurements of the long-slit data: knots $\mathrm{B}$ and $\mathrm{C}$.

\begin{tabular}{|c|c|c|}
\hline & B & $\mathrm{C}$ \\
\hline$n([\mathrm{~S} \mathrm{II}])$ & 100: & 100: \\
\hline$t_{\mathrm{e}}([\mathrm{O} \mathrm{III}])$ & $1.26 \pm 0.03$ & $1.01 \pm 0.02^{c}$ \\
\hline$t_{\mathrm{e}}\left(\left[\mathrm{S}_{\mathrm{III}}\right]\right)$ & $1.19 \pm 0.12$ & $0.88 \pm 0.13^{d e}$ \\
\hline$t_{\mathrm{e}}([\mathrm{O} \mathrm{II}])$ & $1.20 \pm 0.04$ & $1.21 \pm 0.01^{a}$ \\
\hline$t_{\mathrm{e}}\left(\left[\mathrm{S}_{\mathrm{II}}\right]\right)$ & $0.79 \pm 0.11$ & $1.21 \pm 0.01^{a}$ \\
\hline$t_{\mathrm{e}}([\mathrm{N}$ II $])$ & $1.20 \pm 0.04^{a}$ & $0.93 \pm 0.01^{e}$ \\
\hline $12+\log \left(\mathrm{O}^{+} / \mathrm{H}^{+}\right)$ & $7.34 \pm 0.05$ & $7.82 \pm 0.06^{a}$ \\
\hline $12+\log \left(\mathrm{O}^{2+} / \mathrm{H}^{+}\right)$ & $7.95 \pm 0.03$ & $7.84 \pm 0.03^{c}$ \\
\hline $12+\log (\mathrm{O} / \mathrm{H})$ & $8.04 \pm 0.03$ & $8.13 \pm 0.04^{b}$ \\
\hline $12+\log \left(\mathrm{S}^{+} / \mathrm{H}^{+}\right)$ & $5.95 \pm 0.20$ & $6.16 \pm 0.07^{a}$ \\
\hline $12+\log \left(\mathrm{S}^{2+} / \mathrm{H}^{+}\right)$ & $6.17 \pm 0.23$ & - \\
\hline $\mathrm{ICF}\left(\mathrm{S}^{+}+\mathrm{S}^{2+}\right)$ & $1.41 \pm 0.03$ & - \\
\hline $12+\log (\mathrm{S} / \mathrm{H})$ & $6.52 \pm 0.22$ & - \\
\hline $\log (\mathrm{S} / \mathrm{O})$ & $-1.52 \pm 0.22$ & - \\
\hline $12+\log \left(\mathrm{N}^{+} / \mathrm{H}^{+}\right)$ & $6.18 \pm 0.04^{a}$ & $6.70 \pm 0.07^{e}$ \\
\hline $12+\log (\mathrm{N} / \mathrm{H})$ & $6.88 \pm 0.20^{a}$ & $7.02 \pm 0.23^{e}$ \\
\hline $\log (\mathrm{N} / \mathrm{O})$ & $-1.16 \pm 0.07^{a}$ & $-1.11 \pm 0.09^{e}$ \\
\hline $12+\log \left(\mathrm{Ne}^{2+} / \mathrm{H}^{+}\right)$ & $7.33 \pm 0.03$ & - \\
\hline $\mathrm{ICF}\left(\mathrm{Ne}^{2+}\right)$ & $1.08 \pm 0.01$ & - \\
\hline $12+\log (\mathrm{Ne} / \mathrm{H})$ & $7.36 \pm 0.03$ & - \\
\hline $\log (\mathrm{Ne} / \mathrm{O})$ & $-0.68 \pm 0.04$ & - \\
\hline $12+\log \left(\mathrm{Ar}^{2+} / \mathrm{H}^{+}\right)$ & $5.65 \pm 0.10$ & - \\
\hline $12+\log \left(\mathrm{Ar}^{3+} / \mathrm{H}^{+}\right)$ & $4.62 \pm 0.08$ & - \\
\hline $\mathrm{ICF}\left(\mathrm{Ar}^{2+}+\mathrm{Ar}^{3+}\right)$ & $1.03 \pm 0.01$ & - \\
\hline $12+\log (\mathrm{Ar} / \mathrm{H})$ & $5.70 \pm 0.10$ & - \\
\hline $\log (\mathrm{Ar} / \mathrm{O})$ & $-2.34 \pm 0.11$ & - \\
\hline $12+\log \left(\mathrm{Fe}^{2+} / \mathrm{H}^{+}\right)$ & $5.04 \pm 0.10$ & - \\
\hline $\mathrm{ICF}\left(\mathrm{Fe}^{2+}\right)$ & $5.21 \pm 0.62$ & - \\
\hline $12+\log (\mathrm{Fe} / \mathrm{H})$ & $5.76 \pm 0.11$ & - \\
\hline $\mathrm{He}^{+} / \mathrm{H}^{+}(\lambda 4471)$ & $0.064 \pm 0.003$ & - \\
\hline $\mathrm{He}^{+} / \mathrm{H}^{+}(\lambda 5876)$ & $0.096 \pm 0.002$ & - \\
\hline $\mathrm{He}^{+} / \mathrm{H}^{+}(\lambda 6678)$ & $0.072 \pm 0.003$ & - \\
\hline $\mathrm{He}^{+} / \mathrm{H}^{+}(\lambda 7065)$ & $0.100 \pm 0.008$ & - \\
\hline $\mathrm{He}^{+} / \mathrm{H}^{+}$adopted & $0.086 \pm 0.015$ & - \\
\hline $\mathrm{He}^{2+} / \mathrm{H}^{+}(\lambda 4686)$ & $0.0013 \pm 0.0002$ & - \\
\hline$(\mathrm{He} / \mathrm{H})$ & $0.087 \pm 0.015$ & - \\
\hline
\end{tabular}

Note. All the values with a superscript are derived using models or empirical/semi-empirical relations, and the derived errors are underestimated. Densities in $\mathrm{cm}^{-3}$ and temperatures in $10^{4} \mathrm{~K}$.

${ }^{a}$ Derived using temperatures predicted by photoionization models, see the text. ${ }^{b}$ Derived using temperatures estimated from photoionization models and/or empirical methods. ${ }^{c}$ Derived using the H06 empirical method. ${ }^{d}$ Derived using the D07 empirical method. ${ }^{e}$ Derived using the P07 empirical method.

The nitrogen auroral line, $[\mathrm{N} I I] \lambda 5755 \AA$, is unobservable for all the studied star-forming knots. Then, under the same assumption, we estimated this temperature using the approximation $T_{\mathrm{e}}([\mathrm{N}$ II] $])$ $\approx T_{\mathrm{e}}\left(\left[\mathrm{O}_{\text {II }}\right]\right)$, except in the cases where we had to derive the $[\mathrm{N}$ II $]$ temperatures first using Pilyugin's method and estimate the [O II] ones using the same approximation.

(i) The oxygen ionic abundance ratios, $\mathrm{O}^{+} / \mathrm{H}^{+}$and $\mathrm{O}^{2+} / \mathrm{H}^{+}$, were derived from the $\left[\mathrm{O}_{\mathrm{II}}\right] \lambda \lambda 3727,29 \AA$ and [O $\left.\mathrm{III}\right] \lambda \lambda 4959,5007 \AA$ lines, respectively, using for each ion its corresponding tempera- ture. At the temperatures derived for the observed objects, most of the oxygen is in the form of $\mathrm{O}^{+}$and $\mathrm{O}^{2+}$; therefore the approximation $\mathrm{O} / \mathrm{H}=\left(\mathrm{O}^{+}+\mathrm{O}^{2+}\right) / \mathrm{H}^{+}$is valid to represent the total oxygen abundances.

(ii) The sulphur ionic abundances, $\mathrm{S}^{+} / \mathrm{H}^{+}$and $\mathrm{S}^{2+} / \mathrm{H}^{+}$, were derived using $T_{\mathrm{e}}$ ([S $\left.\left.\mathrm{S}_{\mathrm{II}}\right]\right)$ and $T_{\mathrm{e}}\left(\left[\mathrm{S} \mathrm{III}_{\mathrm{II}}\right]\right.$ ), and the fluxes of the $\left[\mathrm{S}_{\mathrm{II}}\right] \lambda \lambda 6717,6731 \AA$ and the near-IR [S III] $\lambda \lambda 9069,9532 \AA$ emission lines, respectively. A relatively important contribution from $\mathrm{S}^{3+}$ may be expected for sulphur, depending on the nebular excitation. Taking into account this unseen ionization state, the total sulphur abundance is calculated using an ICF for $\mathrm{S}^{+}+\mathrm{S}^{2+}$. A good approximation for this ICF in terms of $\mathrm{O}^{+} / \mathrm{O}$ is the formula by Barker (1980), which is based on Stasińska (1978) photoionization models, with $\alpha=2.5$, which gives the best fit to the scarce observational data on $\mathrm{S}^{3+}$ abundances (Pérez-Montero et al. 2006). H08 further reduced the propagated error by writing this ICF in terms of the ratio $\mathrm{O}^{2+} / \mathrm{O}$ instead of $\mathrm{O}^{+} / \mathrm{O}$.

(iii) The nitrogen ionic abundance, $\mathrm{N}^{+} / \mathrm{H}^{+}$, was derived from the intensities of the $\left[\mathrm{N}_{\mathrm{II}}\right] \lambda \lambda 6548,6584$ lines. The N/O abundance ratio was calculated assuming that $\mathrm{N} / \mathrm{O}=\mathrm{N}^{+} / \mathrm{O}^{+}$and $\mathrm{N} / \mathrm{H}$ estimated as $\log (\mathrm{N} / \mathrm{H})=\log (\mathrm{N} / \mathrm{O})+\log (\mathrm{O} / \mathrm{H})$.

(iv) The neon ionic abundance, $\mathrm{Ne}^{2+}$, was derived using the [Ne III] $\lambda 3868 \AA$ emission line, which is the only visible and uncontaminated emission line of this atom in the spectra. For this ion we considered the electron temperature of [O $\mathrm{III}]$, as representative of the high-ionization zone $\left(T_{\mathrm{e}}([\mathrm{Ne} \mathrm{III}]) \approx T_{\mathrm{e}}([\mathrm{O} \mathrm{III}])\right.$; Peimbert \& Costero 1969). Izotov et al. (2004) point out that this assumption can lead to an overestimate of $\mathrm{Ne} / \mathrm{H}$ in objects with low excitation, where the charge transfer between $\mathrm{O}^{2+}$ and $\mathrm{H}^{0}$ becomes important. Thus, we used the expression of this ICF given by Pérez-Montero et al. (2007). Given the high excitation of the observed objects, there are no significant differences between the derived neon abundances using this ICF and those estimated with the classical approximation (H08; H11).

(v) The argon ionic abundance, $\mathrm{Ar}^{2+}$, was calculated from the measured $\left[\mathrm{Ar}\right.$ III] $\lambda 7136 \AA$ emission line assuming that $T_{\mathrm{e}}([\mathrm{Ar} \mathrm{III}])$ $\approx T_{\mathrm{e}}([\mathrm{S} \mathrm{III}])$ (Garnett 1992). In some cases, it is possible to measure the $[\mathrm{Ar}$ IV] $\lambda \lambda 4711,4740 \AA$ emission lines. However, the first one is usually merged with the $\mathrm{He}_{\mathrm{I}} \lambda 4713 \AA$ line, and we are not able to separate them. Therefore, we use the second and more intense line to calculate the $\mathrm{Ar}^{3+}$ abundance. The ionic abundance of $\mathrm{Ar}^{3+}$ was calculated assuming that $T_{\mathrm{e}}([\mathrm{Ar} \mathrm{IV}]) \approx T_{\mathrm{e}}([\mathrm{O} \mathrm{III}])$. The total abundance of argon is hence calculated using the $\operatorname{ICF}\left(\mathrm{Ar}^{2+}\right)$ and the $\mathrm{ICF}\left(\mathrm{Ar}^{2+}+\mathrm{Ar}^{3+}\right)$ derived from photoionization models by Pérez-Montero et al. (2007).

(vi) Finally, for iron used the $[\mathrm{Fe}$ III] $\lambda 4658 \AA$ emission line using the electron temperature of $[\mathrm{O}$ III $]\left[T_{\mathrm{e}}([\mathrm{Fe} \mathrm{III}]) \approx T_{\mathrm{e}}([\mathrm{O}\right.$ III $\left.])\right]$. We took the $\mathrm{ICF}\left(\mathrm{Fe}^{2+}\right)$ from Rodríguez \& Rubin (2004). We were only able to measure the $[\mathrm{Fe} \mathrm{III}] \lambda 4658 \AA$ emission-line flux in the long-slit spectrum of knot B.

The ionic abundances with respect to ionized hydrogen of the elements heavier than helium, ICFs, total abundances and their corresponding errors are also given in Tables 7 and 8 for long-slit and echelle data, respectively.

\section{DISCUSSION}

In the following subsections we will discuss the results obtained from the two different observation modes. 


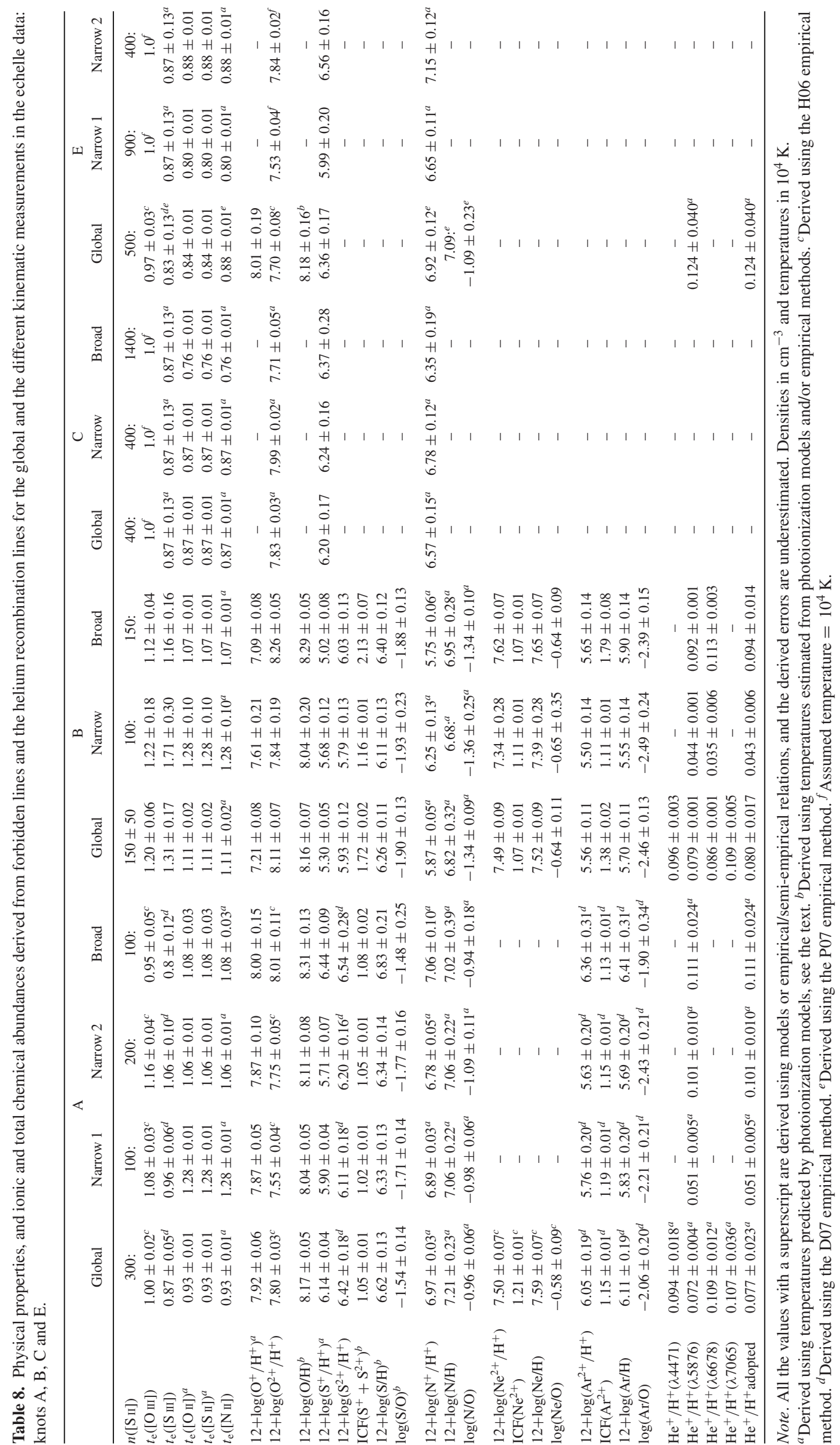



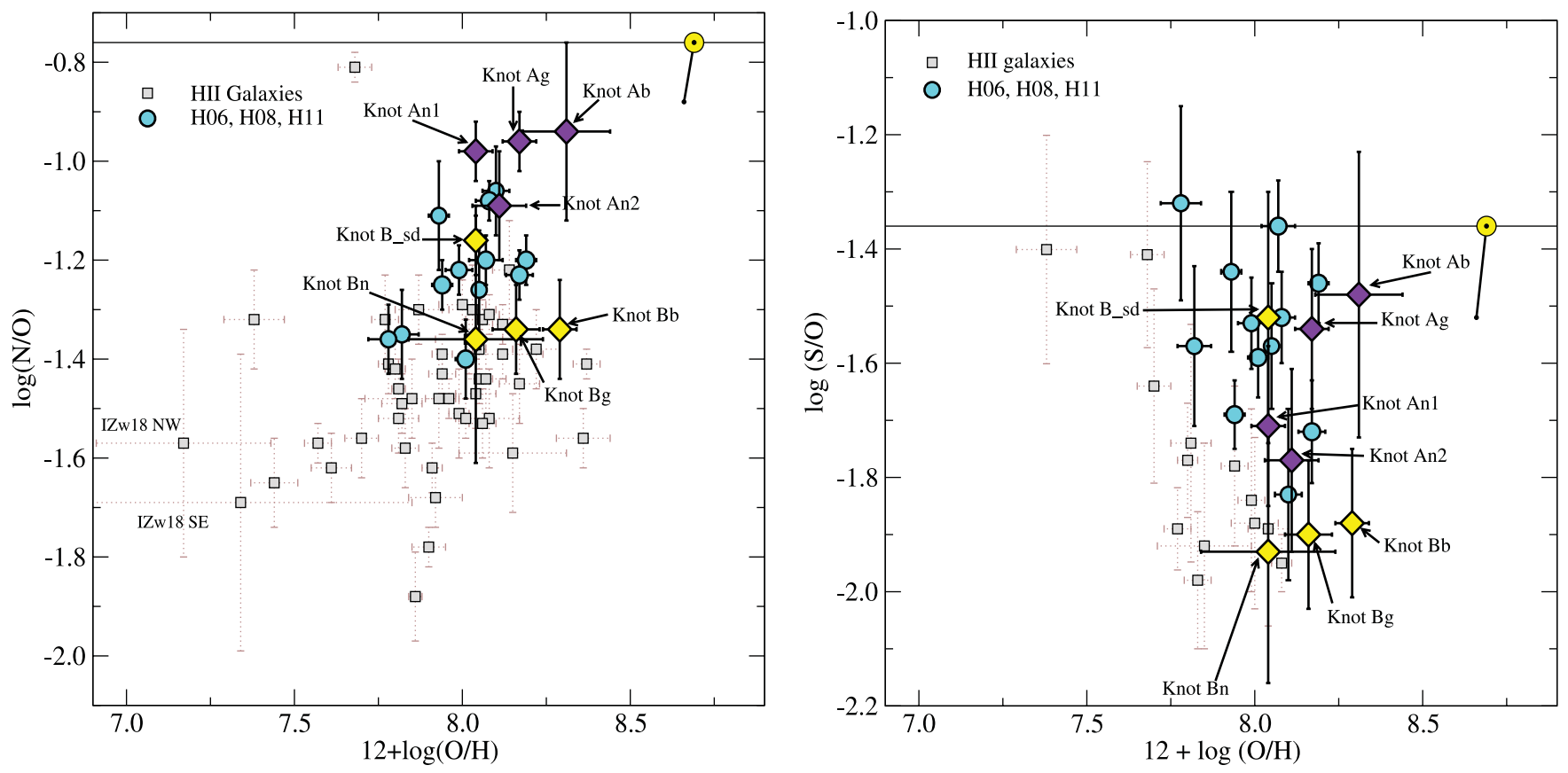

Figure 4. $\mathrm{N} / \mathrm{O}$ and $\mathrm{S} / \mathrm{O}$ as a function of $12+\log (\mathrm{O} / \mathrm{H})$ (left- and right-hand panel, respectively) for knots $\mathrm{A}$ and $\mathrm{B}$ (open and filled diamonds, violet and yellow, respectively), and the objects from $\mathrm{H} 06, \mathrm{H} 08$ and $\mathrm{H} 11$ (open circles, turquoise). The open squares are the H II galaxies from table 13 of H08. The observed knots are marked in the plot with their names plus a letter which denote the observation mode ('sd': single dispersion) or the kinematic component measure (' $\mathrm{g}$ ': global, 'n': narrow and 'b': broad). The solar values are shown with the usual sun symbol: oxygen from Allende Prieto et al. (2001), nitrogen from Holweger (2001) and sulphur from Grevesse \& Sauval (1998). These values are linked by a solid line with the solar ratios from Asplund et al. (2005).

\subsection{Long-slit data}

\section{Haro $15 B$}

Four electron temperatures, $T_{\mathrm{e}}([\mathrm{O} \mathrm{III}]), T_{\mathrm{e}}([\mathrm{O} \mathrm{II}]), T_{\mathrm{e}}([\mathrm{S} \mathrm{III}])$ and $T_{\mathrm{e}}([\mathrm{S}$ II $])$, were estimated from direct measurements for knot B. The good quality of the data translates into small rms errors $(2,3,10$ and 14 per cent, respectively, for each temperature). In the absence of the $[\mathrm{N}$ II] $\lambda 5755 \AA$ emission line we assumed the relationships between temperatures to be $T_{\mathrm{e}}([\mathrm{N}$ II $]) \approx T_{\mathrm{e}}\left(\left[\mathrm{O}_{\text {II }}\right]\right)$.

Knot B shows an electron density value of $n_{\mathrm{e}}=100 \mathrm{~cm}^{-3}$, typical for this kind of objects. However, the error is too large; thus, it should be regarded as an estimation of the order magnitude of the density.

In this knot, we derived the ionic abundances of $\mathrm{O}^{+}, \mathrm{O}^{2+}, \mathrm{S}^{+}$, $\mathrm{S}^{2+}, \mathrm{N}^{+}, \mathrm{Ne}^{2+}, \mathrm{Ar}^{2+}, \mathrm{Ar}^{3+}$ and $\mathrm{Fe}^{2+}$ using the direct method as described in $\mathrm{H} 08$. We also calculated the total abundances of $\mathrm{O}, \mathrm{S}$, $\mathrm{N}, \mathrm{Ne}, \mathrm{Ar}$ and $\mathrm{Fe}$, and the logarithmic ratios of N/O, S/O, Ne/O and $\mathrm{Ar} / \mathrm{O}$.

The oxygen abundance is $8.04 \pm 0.03$, which is 0.22 times the solar value $(12+\log (\mathrm{O} / \mathrm{H}) \odot=8.69$; Allende Prieto, Lambert \& Asplund 2001). This oxygen abundance value is in very good agreement, within the observational errors, with that found by LópezSánchez \& Esteban (2009b), $8.10 \pm 0.06$. The sulphur abundance is 0.15 times the solar value $(12+\log (\mathrm{S} / \mathrm{H}) \odot=7.33$; Grevesse \& Sauval 1998), and the nitrogen abundance is 0.09 times the solar one $\left(12+\log (\mathrm{N} / \mathrm{H})_{\odot}=7.93\right.$; Holweger 2001$)$.

The logarithmic N/O ratio found for knot B is 0.28 dex lower than the solar value $\left(\log (\mathrm{N} / \mathrm{O})_{\odot}=-0.88\right.$; Asplund, Grevesse \& Sauval 2005), and 0.44 dex higher than the typical value shown by this kind of objects which present a plateau at $\log (\mathrm{N} / \mathrm{O})$ about -1.6 dex (Alloin et al. 1979; Pagel et al. 1979) in the low-metallicity end of the distribution (see discussion in Amorín, Pérez-Montero \&
Vílchez 2010; Pérez-Montero et al. 2011, and references therein). The derived value is in the upper range of the distribution of the $\log (\mathrm{N} / \mathrm{O})$ ratio for this kind of objects (see Fig. 4). The $\log (\mathrm{S} / \mathrm{O})$ ratio is $-1.52 \pm 0.22$, in agreement, within the errors, with the solar value $\left(\log (\mathrm{S} / \mathrm{O})_{\odot}=-1.36\right.$; Grevesse \& Sauval 1998) (see Fig. 4). The logarithmic $\mathrm{Ne} / \mathrm{O}$ and $\mathrm{Ar} / \mathrm{O}$ ratios are also consistent with the solar ones $\left(\log (\mathrm{Ne} / \mathrm{O})_{\odot}=-0.61 \mathrm{dex}\right.$ and $\log (\mathrm{Ar} / \mathrm{O})_{\odot}=$ -2.29 dex; Grevesse \& Sauval 1998) within the errors (see Tables 7 and 8).

We used the intensities of the well-detected $\mathrm{He}_{\mathrm{I}} \lambda \lambda 4471,5876$, 6678 and $7065 \AA$, and $\mathrm{He}$ II $\lambda 4686 \AA$ lines to estimate the ionic abundances of $\mathrm{He}^{+}$and $\mathrm{He}^{2+}$, respectively. Then we derived the helium total abundance using these ionic abundances. The adopted value for $\mathrm{He}^{+} / \mathrm{H}^{+}$is $0.086 \pm 0.015$ and for $\mathrm{He}^{2+} / \mathrm{H}^{+} 0.0013 \pm$ 0.0002 . The He total abundance estimated in this region is $0.087 \pm$ 0.015 , within the typical values found for $\mathrm{H}$ II galaxies (see e.g. H08, and references therein).

\section{Haro $15 C$}

In the case of knot $\mathrm{C}$ it was impossible to obtain any electron temperature using the direct method. In this case, we estimated the temperatures using the models and the empirical relationships, as explained in the previous section.

The auroral lines are not detected in the knot $\mathrm{C}$ spectrum due to the low $\mathrm{S} / \mathrm{N}$ of these data, so we estimated a $\left[\mathrm{N}_{\mathrm{II}}\right]$ temperature of $9300 \pm 100 \mathrm{~K}$ following Pilyugin's method (P07). Using the approximation $T_{\mathrm{e}}([\mathrm{N}$ II $]) \approx 500 \mathrm{~K}+T_{\mathrm{e}}([\mathrm{S} \mathrm{III}])$ derived by D07, we estimated $T_{\mathrm{e}}([\mathrm{S} \mathrm{III}])$, and derived $T_{\mathrm{e}}([\mathrm{O} \mathrm{III}])$ from the empirical relation fitted by $\mathrm{H} 06$. The electron temperature $T_{\mathrm{e}}([\mathrm{O} \mathrm{II}])$ was calculated from $T_{\mathrm{e}}([\mathrm{O} \mathrm{III}])$ using the relationship based on photoionization models derived by Pérez-Montero \& Díaz (2003), and from 
the approximation $T_{\mathrm{e}}([\mathrm{S} \mathrm{II}]) \approx T_{\mathrm{e}}\left(\left[\mathrm{O}_{\mathrm{II}}\right]\right)$ we obtained the electron temperature of $[\mathrm{S} \mathrm{II}]$. As in the case of knot $\mathrm{B}$, due to the large errors involved, the derived electron density of 100 particles $\mathrm{cm}^{-3}$ has to be regarded as a rough estimation.

In this knot, we were not able to measure the [Fe III] $\lambda 4658$ emission line and therefore no iron abundance could be derived. The $\left[\mathrm{N}_{\text {II }}\right] \lambda 6548 \AA$ emission line could not be measured either and was taken to be 0.33 times that of $\left[\mathrm{N}_{\mathrm{II}}\right] \lambda 6584 \AA$.

\section{Comparison among knots}

A comparison between the results for both knots from long-slit data might suggest, at face value, that the oxygen abundance could be slightly higher in knot C. However, the $1 \sigma$ errors derived for these oxygen abundances do not take into account the errors in the line temperatures introduced by uncertainties in the calibrations of the relations based on photoionization models. These unaccounted effect will most probably make the observed difference negligible. The estimated N/O ratio for knot $\mathrm{C}$ is only marginally larger than that for knot B, but still the same within the observational errors.

The temperatures representative of the high-ionization zone are higher for knot $\mathrm{B}$ than for knot $\mathrm{C}$. The temperatures for the lowionization zone show a different behaviour: $T_{\mathrm{e}}\left(\left[\mathrm{O}_{\mathrm{II}}\right]\right)$ appear to be similar in both knots while the $T_{\mathrm{e}}([\mathrm{S}$ II] $])$ in knot B is $4200 \mathrm{~K}$ lower than that in knot $\mathrm{C}$. We have to note that the $\left[\mathrm{S}_{\mathrm{II}}\right]$ temperature was derived using the direct method for knot $\mathrm{B}$, but using photoionization models for knot $\mathrm{C}$. This leads to a difference in temperature among [O II] and [S II] for knot B, although models state that we should expect similar values. This behaviour was previously found for several objects (see fig. 2.17 of Hägele 2008). Moreover, PérezMontero et al. (2010) presented a self-consistent study in a sample of $10 \mathrm{H}$ II galaxies computing tailored models with the photoionization code CLOUDY (Ferland et al. 1998). They found that the electron temperature of $[\mathrm{S} \mathrm{II}]$ was overestimated by the models, with the corresponding underestimate of its abundance, pointing to the possible presence of outer shells of diffuse gas in these objects that were not taken into account in their models. In contrast, the $[\mathrm{N}$ II] temperature for knot B is higher than that derived for knot $\mathrm{C}$ by about $2700 \mathrm{~K}$, but again they were estimated using two different methods. Only for comparison purposes we also estimated this temperature for knot B using Pilyugin's method finding $T_{\mathrm{e}}\left(\left[\mathrm{N}_{\mathrm{II}}\right]\right)=10500 \pm 100 \mathrm{~K}$, which is lower than the estimated using the models by $1500 \mathrm{~K}$. Comparing those $[\mathrm{N}$ II] temperatures derived using Pilyugin's method, knot B still shows higher values than knot $\mathrm{C}$ by about $1200 \mathrm{~K}$.

The excitation degree, given by the logarithmic $\mathrm{O}^{2+} / \mathrm{O}^{+}$ratio, is higher in knot $\mathrm{B}(0.61 \mathrm{dex})$ than in knot $\mathrm{C}$ (0.02 dex), in agreement with the estimated values by López-Sánchez \& Esteban (2009a). The estimated extinction in both knots is small, typical of this kind of objects (Hägele 2008, and references therein).

\subsection{Echelle data}

We analysed the parameters derived from the global and the different kinematical component measurements for the four knots using the echelle data.

\section{Haro 15 A}

A large number of recombination and forbidden lines were detected in our echelle spectrum, although we were not able to measure any auroral emission line. We therefore used the relation between the $\mathrm{SO}_{23}$ parameter and the [S III] temperature derived by D07 to estimate $T_{\mathrm{e}}([\mathrm{S} \mathrm{III}])$. From this temperature we calculated $T_{\mathrm{e}}[\mathrm{O} \mathrm{III}]$ using the relationship found by H06. Following the classical analysis (see $\mathrm{H} 08)$, we estimated $T_{\mathrm{e}}([\mathrm{O} \Pi]), T_{\mathrm{e}}([\mathrm{S} \mathrm{II}])$ and $T_{\mathrm{e}}([\mathrm{N}$ II] $])$ using the relations given by photoionization models.

The estimated density errors are large; hence, these values are only considered as an order of magnitude. In all cases, the electron densities are well below the critical value for collisional deexcitation.

The global measure presents a total oxygen abundance of $8.17 \pm$ $0.05,0.3$ times the solar value, while for the different kinematical components we obtained $8.04 \pm 0.05$ (narrow 1), $8.11 \pm 0.08$ (narrow 2) and $8.31 \pm 0.13$ (broad), 0.22, 0.26 and 0.42 times the solar abundance, respectively. There seems to be a difference between broad and narrow components above $1 \sigma$ level, but since the errors in the calibration of the relations based on photoionization models are not quantitatively established, we can consider the total abundances in agreement for all components. The derived total oxygen abundances for the different kinematical components and for the global measure in this knot present the characteristic low values, within the errors, that are found for $\mathrm{H}$ II galaxies: $12+\log (\mathrm{O} / \mathrm{H})$ between 7.94 and 8.19 (Terlevich et al. 1991; Hoyos \& Díaz 2006).

Taking the errors into account, the total sulphur abundance of the broad component is about 0.15 dex higher than those shown by the narrow ones, but in agreement considering errors at $2 \sigma$ level. On the other hand, the values for total nitrogen abundances are in very good agreement for all the components.

The ionic abundances of sulphur, $\mathrm{S}^{+} / \mathrm{H}^{+}$and $\mathrm{S}^{2+} / \mathrm{H}^{+}$, the ionic nitrogen abundance, $\mathrm{N}^{+} / \mathrm{H}^{+}$, and the ionic argon abundance, $\mathrm{Ar}^{2+} / \mathrm{H}^{+}$, obtained for the broad component are higher than the ionic abundances found for the narrow components (between 0.17 and 0.61 dex with respect to the narrow 1 component, and between 0.28 and 0.73 dex with respect to the narrow 2 component). Only for the global measure it was possible to obtain the ionic and total abundances of $[\mathrm{Ne} \mathrm{III}]$ from the measure of the neon $3868 \AA$ emission line.

We also derived the sulphur and nitrogen abundances relative to the oxygen abundance, $\mathrm{S} / \mathrm{O}$ and N/O. If we consider the errors, all the S/O derived values are very similar among them (see Fig. 4). The $\mathrm{N} / \mathrm{O}$ value estimated for the narrow 2 component is the lowest, but its difference with the other components is negligible. This behaviour of the N/O could be evidence of a common or very similar chemical evolution for the different kinematical components (see discussion in Pérez-Montero et al. 2011). It could also be indicative that the different kinematical components correspond to different phases of the same gas, which is in agreement with the scenarios proposed, for example, by Tenorio-Tagle, Munoz-Tunon \& Cid-Fernandes (1996) or Westmoquette et al. (2009). In knot A we also obtained an excess of the N/O ratio (see Fig. 4) with respect to the typical values found for an $\mathrm{H}$ II galaxy.

For the global measure we can determine the ionic helium abundance, from the 4471, 5876, 6678, $7065 \AA$ emission lines. For the three kinematical components we were only able to deconvolve the $5876 \AA$ emission line. The narrow 1 component has a lower ionic abundance than the typical values found for other similar galaxies, while the values found for the other two components and the global measure are in the range presented by $\mathrm{H}$ II galaxies (see $\mathrm{H} 08$ ).

\section{Haro $15 B$}

In this knot we were able to estimate $T_{\mathrm{e}}\left(\left[\mathrm{O}_{\mathrm{III}}\right]\right)$ and $T_{\mathrm{e}}\left(\left[\mathrm{S}_{\mathrm{III}}\right]\right)$ using the direct method through the deconvolution of the emission 
profile for the different kinematical components of the $\left[\mathrm{O}_{\text {III }}\right] \lambda 4363 \AA$ and $\left[\mathrm{S}_{\text {III }}\right] \lambda 6312 \AA$ auroral emission lines. The precision obtained for $T_{\mathrm{e}}([\mathrm{O} \mathrm{III}])$ and $T_{\mathrm{e}}([\mathrm{S} \mathrm{III}])$ is of the order of 5 and 13 per cent for the global measure, 15 and 18 per cent for the narrow component and 4 and 14 per cent for the broad component, respectively, for each temperature. These are the only two temperatures directly derived, even for the global measure. The [O II] temperature was obtained from $T_{\mathrm{e}}([\mathrm{O} \mathrm{III}])$ using the relations given by photoionization models (Pérez-Montero \& Díaz 2003). The [S II] and $[\mathrm{N}$ II] temperatures were estimated using the approximation $T_{\mathrm{e}}([\mathrm{S}$ II $]) \approx T_{\mathrm{e}}([\mathrm{N} \mathrm{II}]) \approx T_{\mathrm{e}}([\mathrm{O}$ II $])$.

The errors in the electron densities are large enough so as to consider our estimate as an order of magnitude. Once again, in all cases the electron densities are well below the critical value for collisional de-excitation.

The ionic abundances in the low-ionization zone $\left(\mathrm{O}^{+} / \mathrm{H}^{+}, \mathrm{S}^{+} / \mathrm{H}^{+}\right.$, $\mathrm{N}^{+} / \mathrm{H}^{+}$) derived for the narrow component are higher than those shown by the broad one, while the ions in the medium- and highionization zones $\left(\mathrm{S}^{2+} / \mathrm{H}^{+}\right.$and $\mathrm{Ar}^{2+} / \mathrm{H}^{+}$, and $\mathrm{O}^{2+} / \mathrm{H}^{+}$and $\mathrm{Ne}^{2+} / \mathrm{H}^{+}$, respectively) show the opposite behaviour, i.e. lower values for the narrow component.

The total oxygen abundances derived for the global measure and for the kinematical components are in good agreement taking into account the observational errors, where the global measure is approximately equal to the average of the derived values for the different kinematical components weighted by luminosity. The values of the total sulphur abundances derived for both kinematical components are very similar, with the broad component presenting slightly larger values than the narrow one. In the case of the total nitrogen abundance we derived very similar values for the different components, although we have to note that the calculated error for the narrow component is too large, and therefore this is only a rough estimate of the nitrogen abundance. The total abundances of $\mathrm{Ne}$ derived for the different components are in agreement within the errors, and the Ar total abundance from the broad component is slightly larger (by about $0.07 \mathrm{dex}$ ) than that derived for the narrow one. As we said above, the temperatures estimated using the relationships based on photoionization models only take into account the errors of the line intensity measurements, but without assigning any errors to the relation parameters. We could therefore state that all the components present total abundances in overall good agreement.

The $\mathrm{S}, \mathrm{N}, \mathrm{Ne}$ and Ar abundances relative to oxygen for the narrow and broad components present mainly the same values, even comparing these values with those estimated for the global measure (see Fig. 4). As in the case of knot A, this could be indicative that both components have a common or very similar chemical evolution and/or that both components represent two different phases of the same gas.

From the global measure, we derived the ionic abundance of once ionized helium using the same emission lines as for knot A. The adopted abundance value is in good agreement with what we find in the long-slit spectrum for the same knot, and in the range found for other $\mathrm{H}$ II galaxies (see H08). We were able to deconvolve the profiles of $\mathrm{He}_{\mathrm{I}} \lambda \lambda 5876$ and $6678 \AA$ emission lines finding very low values for the narrow component, while the broad component presents values typical for this class of objects.

\section{Haro $15 C$ and Haro 15 E}

In the cases of the star-forming knots $\mathrm{C}$ and $\mathrm{E}$, we were not able to detect any auroral emission line. Owing to the low $\mathrm{S} / \mathrm{N}$ of these spectra, other some important and relatively strong emission lines, such as $[\mathrm{O}$ II $] \lambda 3727 \AA$, do not have enough intensity to be measured, even for the global measure. For all the components of knot $\mathrm{C}$ and the kinematical components of knot $\mathrm{E}$, we were not able to measure the emission lines needed to apply the relationships given by $\mathrm{D} 07$ and $\mathrm{P} 07$ to derive the $[\mathrm{S} \mathrm{III}]$ and $[\mathrm{N} \mathrm{II}]$ line temperatures, respectively. We assumed that $T_{\mathrm{e}}([\mathrm{O} \mathrm{III}])$ is equal to $10^{4} \mathrm{~K}$ (as was discussed in Section 3.2); then we estimated the [O II] temperature in each case using its relation with $T_{\mathrm{e}}([\mathrm{O} \mathrm{III}])$ given by photoinization models (Pérez-Montero \& Díaz 2003). $T_{\mathrm{e}}([\mathrm{S}$ II] $])$ and $T_{\mathrm{e}}([\mathrm{N}$ II] $]$ ) were assumed to be equal to the [O II] temperature, and $T_{\mathrm{e}}([\mathrm{S}$ III] $)$ was determined using its relationship with the temperature of [O $\mathrm{III}]$ found by $\mathrm{H} 06$. For the global measure of knot $\mathrm{E}$, it was possible to measure the $\left[\mathrm{O}_{\mathrm{II}}\right] \lambda \lambda 3727,29 \AA$ emission lines. Using these emission lines together with the strong [O III] lines, we applied Pilyugin's method to estimate the electron temperature of [N II]. Using the systematic difference of $500 \mathrm{~K}$ between the temperatures of [N II] and [S III] found by D07, we estimated $T_{\mathrm{e}}([\mathrm{S} \mathrm{III}])$. From the $[\mathrm{S}$ III] temperature we derived $T_{\mathrm{e}}([\mathrm{O} \mathrm{III}])$ using the relation by $\mathrm{H} 06$; then we used the same relations as for the kinematical components of knot $\mathrm{E}$ to derive $T_{\mathrm{e}}([\mathrm{O} \mathrm{II}])$ and $T_{\mathrm{e}}([\mathrm{S} \mathrm{II}])$.

The estimated electron densities for these two star-forming knots are rather higher than those found for knots A and B. Due to the very large uncertainties present in all the cases, these density values are only estimates of the order of magnitude of the electron densities. Only for the global measure of knot E, it was possible to obtain an estimate of the ionic abundance of $\mathrm{O}^{+}$and, thus, the total abundances of oxygen and nitrogen, and the N/O ratio.

In contrast with the behaviour found for the $\mathrm{O}^{2+} / \mathrm{H}^{+}$ionic abundance for the star-forming knots $\mathrm{A}$ and $\mathrm{B}$, the ionic abundance of the narrow component for knot $\mathrm{C}$ is higher $(\sim 0.3 \mathrm{dex})$ than the one derived for the broad component. This effect could be due to a different ionization degree of the kinematical components. In the case of knot $\mathrm{E}$, the total oxygen abundance for the global measure is similar to the abundances derived for knots $\mathrm{A}$ and $\mathrm{B}$, and the helium abundance is slightly larger than that found for the other knots.

\section{Comparison among knots}

The total oxygen abundance derived for the global measure of knot A is slightly lower than the value obtained by López-Sánchez \& Esteban $(2009 b ; 8.37 \pm 0.10)$. It could be due to the different hypotheses considered to determine the electron temperatures and the chemical abundances. The total oxygen abundance estimated from the global measure for knot B is in very good agreement with the value obtained by López-Sánchez \& Esteban $(8.10 \pm 0.06)$ for the same knot. We estimated its electron temperature of [O $\mathrm{III}]$ using the direct method, and it is in very good agreement with the value derived by López-Sánchez \& Esteban (12900 \pm 700 K). Our estimate of $T_{\mathrm{e}}([\mathrm{O} \mathrm{II}])$ is in agreement, although slightly lower, with the value derived by López-Sánchez \& Esteban $(12000 \pm 500 \mathrm{~K})$ using the relation given by Garnett (1992).

Recent studies in this galaxy suggest that the ionizing star cluster in knot B would have the youngest population of the galaxy ( $~ 100$ Myr). This knot presents a blue colour and high UV emission, indicating a recent star formation activity, supported by the presence of Wolf-Rayet features in its spectrum (López-Sánchez \& Esteban 2010a). This is in agreement with our measurements of the equivalent width of the $\mathrm{H} \beta$ emission line $[\mathrm{EW}(\mathrm{H} \beta)]$ (see Tables 2 6 ), which suggest that knot $\mathrm{B}$ is the youngest one since it has the highest $\mathrm{EW}(\mathrm{H} \beta)$ (Terlevich et al. 2004). 
On the other hand, we can assume from the analysis performed that the total oxygen abundances derived for knots $\mathrm{A}$ and $\mathrm{B}$ are very similar. The ionic oxygen abundances of $\mathrm{O}^{+} / \mathrm{H}^{+}$derived for knot $\mathrm{B}$ using the long-slit spectrum and the global measure of the echelle data are in good agreement taking into account the errors involved. Under the same considerations, we can assume that the differences between the $\mathrm{O}^{2+} / \mathrm{H}^{+}$ionic abundances and the total oxygen abundances derived for the long-slit and for the global measure of the echelle spectrum of knot $\mathrm{B}$ are negligible. The total oxygen abundances derived from both instruments and from different components for knots A, B, C and E are in good agreement taking into account the observational errors.

The differences found between these star-forming knots of Haro 15 are similar (or even smaller) to what is found in other works which spatially resolve individual knots that belong to $\mathrm{H}$ II galaxies or BCD galaxies (see e.g. Kehrig et al. 2008; Cairós et al. 2009; Pérez-Montero et al. 2009, 2011; García-Benito et al. 2010; $\mathrm{H} 11)$. However, in general, these differences were attributed to the observational uncertainties (pointing errors, seeing variations, etc.) or errors associated with the reddening correction and flux calibration. The oxygen abundance variations were therefore not assumed as statistically significant, concluding that there is a possible common chemical evolution scenario in all of them. There are even greater differences when comparing the estimated abundances of the individual knots with those derived from the integrated spectra of the galaxies. For instance, Cairós et al. (2009) found for the integrated spectrum of Mrk 1418 a lower value of the direct oxygen abundance by about 0.35 dex (equivalent to a factor of 2.2) than for knots 1 and 2 of that galaxy. They pointed out that while this variation could reflect a real abundance difference in different scales (kpc-sized aperture for the integrated spectrum and sizes of the order of $100 \mathrm{pc}$ for individual $\mathrm{H}$ II regions), it may also be due to relatively large measurement uncertainties for the weak [O III] auroral emission line. Fortunately, as in H11 (where we used a double-beam long-slit spectrograph), our data are not affected by pointing errors and seeing variations, and the other observational uncertainties have a second-order effect, since the echelle spectrograph simultaneously acquires all the observed spectral range. Likewise, the errors associated with the measurements of the weak auroral emission lines are relatively small, specially for [O III].

The estimated extinction for all the star-forming knots analysed using different instruments and kinematical components (including the global measure) is very similar and consistent with low extinction values, found in this kind of objects (Hägele 2008, and references therein).

\subsection{Ionization structure}

Vílchez \& Pagel (1988) showed that the quotient of $\mathrm{O}^{+} / \mathrm{O}^{2+}$ and $\mathrm{S}^{+} / \mathrm{S}^{2+}$, called the 'softness parameter' and denoted by $\eta$, is intrinsically related to the shape of the ionizing continuum and depends only slightly on the geometry. We can perform an analysis of the ionization structure only for knots A and B since we need information about two consecutive ionization stages of two different atoms, reliably detected only in these two star-forming knots.

The purely observational counterpart of the $\eta$ parameter is the $\eta^{\prime}$ parameter defined by Vílchez \& Pagel (1988) as the ratio between $\left[\mathrm{O}_{\mathrm{II}}\right] /\left[\mathrm{O}_{\mathrm{III}}\right]$ and $\left[\mathrm{S}_{\mathrm{II}}\right] /[\mathrm{S} \mathrm{III}]$. The relation between both parameters is

$\log \eta^{\prime}=\log \eta-0.14 t_{\mathrm{e}}-0.16$,

where $t_{\mathrm{e}}$ is the electron temperature in units of $10^{4} \mathrm{~K}$.

The left-hand panel of Fig. 5 shows the relation between $\log \left(\mathrm{O}^{+} / \mathrm{O}^{2+}\right)$ and $\log \left(\mathrm{S}^{+} / \mathrm{S}^{2+}\right)$ derived for knots $\mathrm{A}$ and $\mathrm{B}$. The global measures and the different kinematical components of knot $\mathrm{A}$ and $\mathrm{B}$ are represented with violet and yellow diamonds, respectively. In
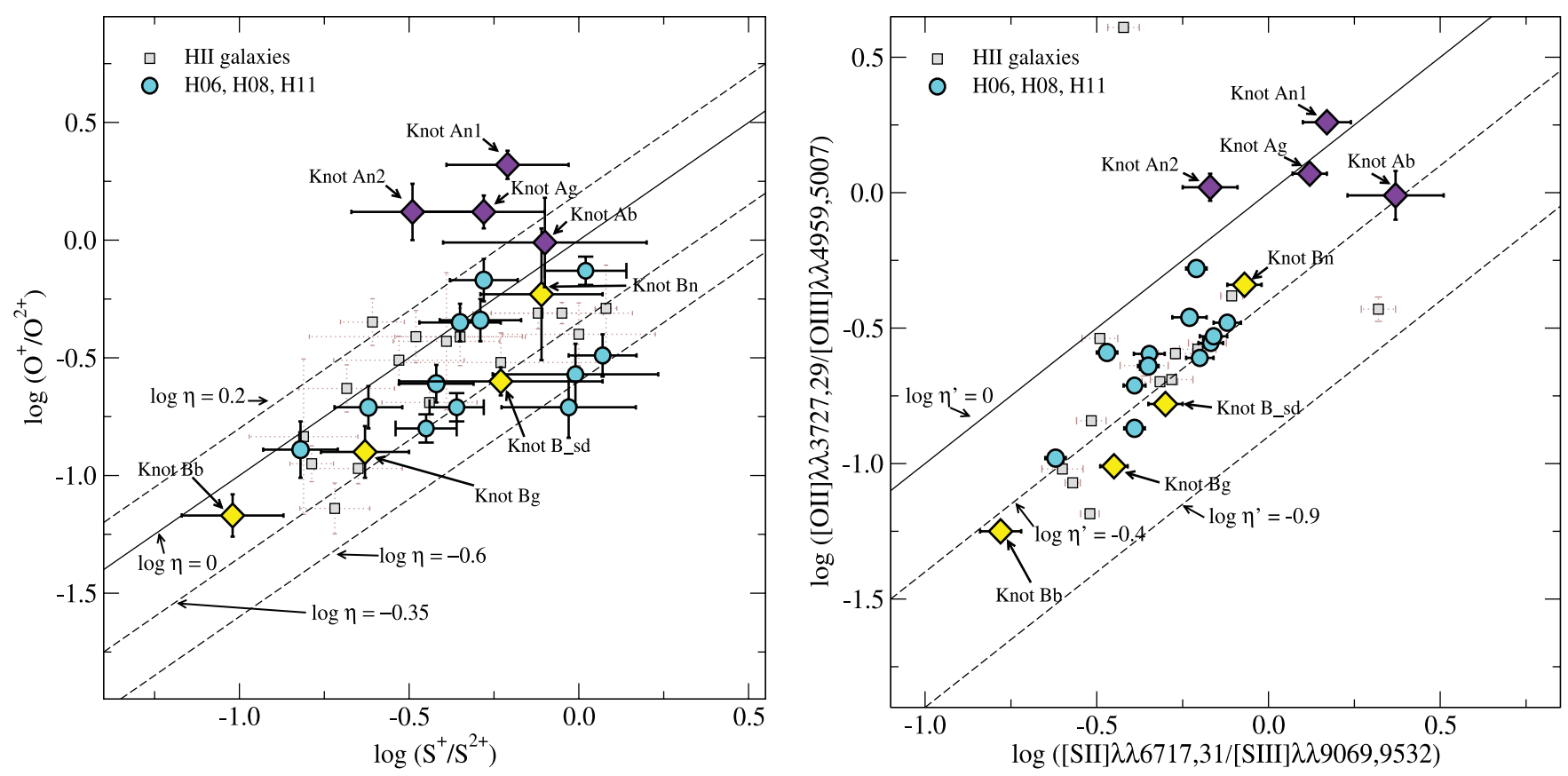

Figure 5. Left-hand panel: $\log \left(\mathrm{O}^{+} / \mathrm{O}^{2+}\right)$ versus $\log \left(\mathrm{S}^{+} / \mathrm{S}^{2+}\right)$ for knots A and B (filled violet and yellow diamonds, respectively), the objects studied in H06, $\mathrm{H} 08$ and $\mathrm{H} 11$ (turquoise circles), and $\mathrm{H}$ II galaxies from the literature as described in H08 (open squares). The diagonals in this diagram correspond to constant values of $\eta$. Right-hand panel: $\log \left(\left[\mathrm{O}_{\mathrm{II}}\right] /[\mathrm{O} \mathrm{III}]\right)$ versus $\log \left(\left[\mathrm{S}_{\mathrm{II}}\right] /\left[\mathrm{S}_{\mathrm{III}}\right]\right)$, symbols as in the left-hand panel. The diagonals in this diagram correspond to constant values of $\eta^{\prime}$. As in Fig. 4, the letter added to the name of each knot denotes the instrument (for the long-slit spectrum) or the components (for echelle data). 
the right-hand panel we show the relation between $\log \left(\left[\mathrm{O}_{\mathrm{II}}\right] /[\mathrm{O} \mathrm{III}]\right)$ and $\log \left(\left[\mathrm{S}_{\mathrm{II}}\right] /[\mathrm{S} \mathrm{III}]\right)$, which does not require the explicit knowledge of the involved line temperatures for the derivation of ionic ratios and is independent of the methods used to estimate the line temperatures. The objects studied by H06, H08 and $\mathrm{H} 11$ are represented with turquoise circles, and the $\mathrm{H}_{\text {II }}$ galaxies from the literature (see description and references in H08) with squares. The $\mathrm{H}_{\text {II }}$ galaxies are located in the region corresponding to high values of the effective temperatures of the radiation field of the ionizing star cluster, where $\log \eta$ are between -0.35 and 0.2 (see H06). In Fig. 5, the diagonal lines represent constant values of $\log \eta$ (left-hand panel) and $\log \eta^{\prime}$ (right-hand panel).

The ionization structures derived from both diagrams, $\eta$ and $\eta^{\prime}$, for all the measurements (both instruments and different components) of knot B are almost equal, showing almost the same values within the observational errors. This implies that the effective temperatures of the radiation fields that ionize the gas are very similar for different kinematical components; then the ionizing star clusters that excite the gas that produces the different kinematical components could be the same. For knot A we also derived very similar results from both diagrams. In this case the broad kinematical component shows an ionization structure slightly displaced in the $\eta^{\prime}$ diagram towards higher effective temperature of the ionizing radiation field than those shown by both narrow kinematical components (and the global measure). However, all the components are almost in the same zone in the $\eta$ diagram if we take into account the large observational errors. This fact points again towards a common star cluster as the ionizing source for all the components of knot A. The difference in the ionizing structure of these two knots suggests a different evolutionary stage, since all the components of knot A seem to be located in a region with lower effective temperature of the ionizing radiation field than that where the components of knot $\mathrm{B}$ are placed. This is in agreement with the presence of an older and more evolved ionizing star cluster in knot A than in knot B, as suggested by López-Sánchez \& Esteban (2010a).

\subsection{Chemical abundances from empirical calibrators}

Different strong-line empirical metallicity calibrators are commonly used to estimate the oxygen abundances in objects for which direct derivation of electron temperatures is not feasible. These empirical methods are based on the cooling properties of ionized nebulae which ultimately translate into a relationship between emission-line intensities and oxygen abundance. These relationships have been widely studied in the literature using different strong-line empirical methods which are based on calibrations of the relative intensity of some bright emission lines against the abundance of relevant ions present in the nebula (see e.g. García-Lorenzo et al. 2008; Cairós et al. 2009; García-Benito 2009; García-Benito et al. 2010).

In Fig. 6, we show the total oxygen abundances as derived from several strong-line empirical methods, with their corresponding errors estimated taking into account the errors of the line intensities and also the errors given by the calibrations of the empirical parameters. We also plotted the total oxygen abundances calculated from the electron temperatures measured using the direct method, only for the long-slit data of knot B, and those estimated from the photoionization models and empirical temperature relations. The colours of these non-continuous lines (see the electron edition of the journal) correspond to each measure or component; thus, the black solid and double- dash-dotted lines represent the total oxygen abundances calculated from the long-slit data, and turquoise dash-dotted (green dashed) and magenta dotted lines represent the narrow [narrow 1 (turquoise) and narrow 2 (green) for knot A and E] and broad components from the echelle data, respectively. For simplicity, the abundance ranges corresponding to the global measure for knots A and B are not shown. This value is plotted only for knot $\mathrm{E}$ since it is the only previous oxygen abundance estimation for this knot.

Among the available strong-line empirical parameters to obtain the total oxygen abundance we studied the $S_{23}$ (Vílchez \& Esteban 1996) using the calibration obtained by Pérez-Montero \& Díaz (2005); $\mathrm{O}_{23}$ (Pagel et al. 1979), and according to the values measured in our data, we used the McGaugh (1991) calibration; $\mathrm{SO}_{23}$ ( = $\mathrm{S}_{23} / \mathrm{O}_{23}$; Díaz \& Pérez-Montero 2000) applying the calibration from Pérez-Montero \& Díaz (2005); $\mathrm{O}_{3} \mathrm{~N}_{2}$ (Alloin et al. 1979) with the calibration established by Pettini \& Pagel (2004); $\mathrm{S}_{3} \mathrm{O}_{3}$ (Stasińska 2006); $\mathrm{N}_{2}$ (Storchi-Bergmann, Calzetti \& Kinney 1994) using the empirical calibration from Denicoló, Terlevich \& Terlevich (2002) and $\mathrm{Ar}_{3} \mathrm{O}_{3}$ (Stasińska 2006).

In the case of knot A, there is good agreement between the total oxygen abundance derived from the global measure using the different parameters and the abundance derived using the temperatures obtained from models. Similar results are found when using empirical temperature relations from the global measure. For the narrow 1 kinematical component there is good agreement in the abundance determination using the empirical parameters: $\mathrm{S}_{23}, \mathrm{O}_{23}$ and $\mathrm{SO}_{23}$, and that estimated using the temperature derived by models and/or empirical temperature relations, while the derived abundance using the other empirical parameters shows small overestimations except the $\mathrm{N}_{2}$ parameter which presents the largest difference. The derived abundance for the narrow 2 component using the empirical parameters is in agreement with that derived using the temperatures obtained from models and/or empirical relations, except in the case of the $\mathrm{N}_{2}$ parameter. The derived abundances from the broad component using different empirical parameters are very similar to that derived using temperatures obtained from models or empirical relations. The exception is the $S_{23}$ parameter that yields an abundance slightly higher.

For the echelle data of knot B, the oxygen abundance calculated for the global measure using the empirical parameters $\mathrm{O}_{23}, \mathrm{O}_{3} \mathrm{~N}_{2}$ and $\mathrm{N}_{2}$ shows a similar value to that derived using the temperatures obtained from models and/or empirical relations. The estimated abundance from the other empirical parameters presents lower values than the abundance derived using the line temperatures. The oxygen abundance for the narrow component derived using the empirical parameters presents similar values to that estimated using the line temperatures, and for the broad component the empirical parameters always present lower values than the one determined using the derived temperatures, except for the $\mathrm{O}_{23}$ that shows a similar estimation. For the single dispersion data, the total oxygen abundance derived from empirical parameters is generally in good agreement with that calculated using the temperature obtained using the direct method. Only the abundance determined using the $\mathrm{SO}_{23}$ empirical parameter is slightly lower than the previously estimated.

For knot $\mathrm{C}$, the total oxygen abundance derived using the empirical parameters is in good agreement with the oxygen abundance value determined using the temperatures derived by models and/or empirical temperatures relations for the long-slit data. For knot $\mathrm{E}$, there is good agreement between the abundances derived from the echelle measurements using the empirical parameters and that estimated using the adopted line temperatures, except for the $\mathrm{N}_{2}$ parameter using the narrow 2 component. 


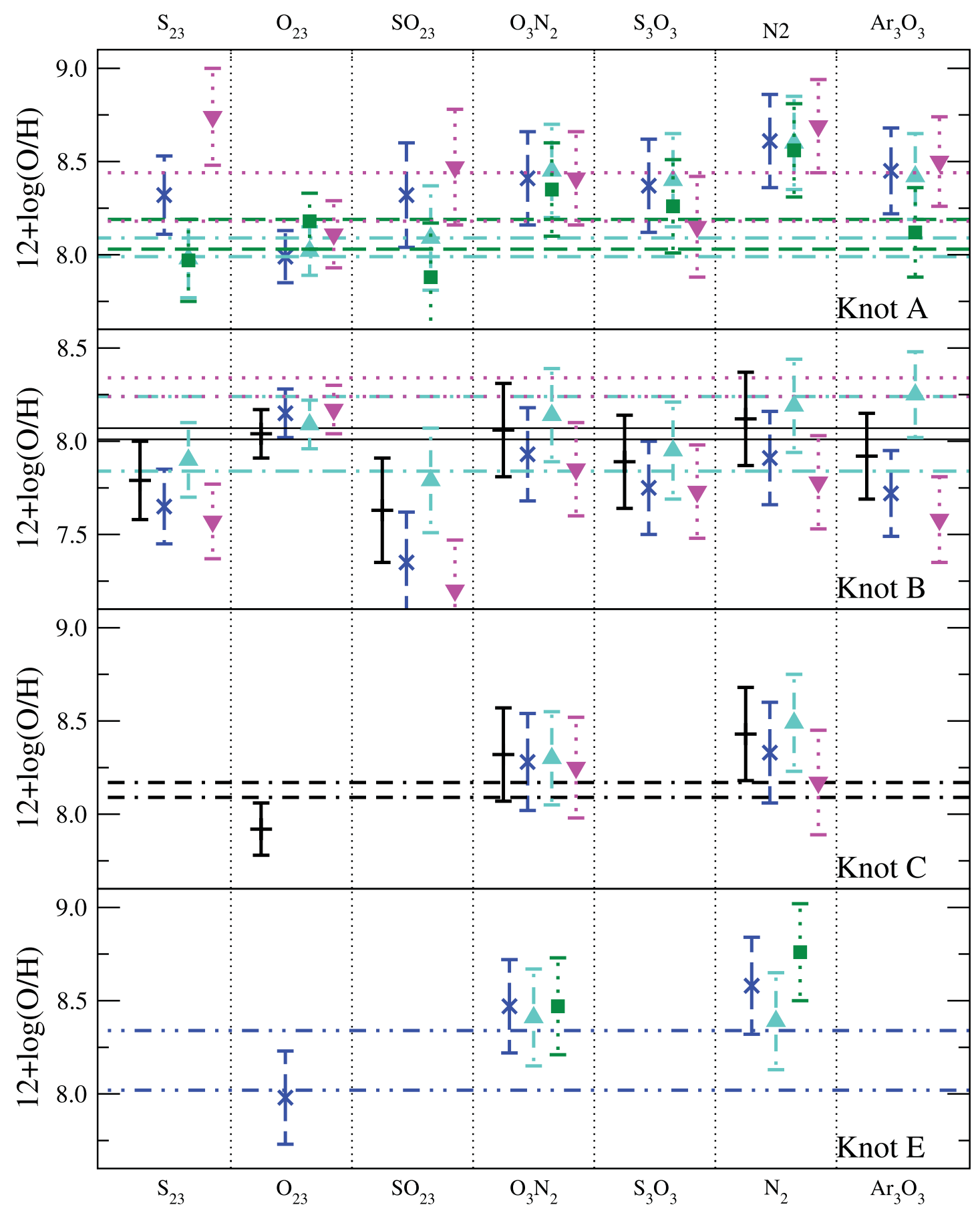

Figure 6. The total oxygen abundances and their uncertainties for both instruments, and the global measure and different kinematical components in each observed knot of Haro 15, as derived using different empirical calibrators. From top to bottom panels: knots A, B, C and E. From left to right: the empirical parameters $\mathrm{S}_{23}, \mathrm{O}_{23}, \mathrm{SO}_{23}, \mathrm{O}_{3} \mathrm{~N}_{2}, \mathrm{~S}_{3} \mathrm{O}_{3}, \mathrm{~N}_{2}$ and $\mathrm{Ar}_{3} \mathrm{O}_{3}$. The solid black error bars represent the long-slit estimate, blue stars: global echelle measure, turquoise up triangles: narrow kinematical component (for knots A and E, filled up triangles: narrow 1 component, and filled green squares: narrow 2 component) and magenta down triangles: broad component. The solid lines represent the valid range taking into account the $1 \sigma$ errors of the total oxygen abundances previously derived using the direct method, and the non-continuous lines the valid range of the total oxygen abundances derived using photoionization models and/or empirical temperature relations (see the text).

We must stress that the different empirical metallicity relationships were derived from global measures of complete regions, and an analysis or calibration for kinematical components has never been done before. To disentangle the empirical relation for these two components, it is mandatory to observe star-forming regions with high spectral resolution, similar to the data presented in our work. Furthermore, even better $\mathrm{S} / \mathrm{N}$ will be needed, in particular to measure the auroral $\mathrm{O}^{+}$emission lines, to be able to obtain direct measurements of the total oxygen abundances for the different kinematical components. It must be noted that empirical relations for 
the estimation of abundances should be applied only in a statistical sense and their application to individual objects or regions is always questionable.

\section{SUMMARY AND CONCLUSIONS}

We have performed an analysis of the characteristics of the ionized gas belonging to the four brightest star-forming knots of the BCD galaxy Haro 15. For two of these regions we present new lowresolution long-slit data covering from 3800 to $9300 \AA$ obtained with the WFCCD camera mounted on the duPont Telescope at LCO. For all the regions we have carried out a chemodynamics study using high-resolution $(R \simeq 25000)$ data acquired with the echelle spectrograph mounted at the same telescope. These highresolution data were previously presented in a kinematical study in Paper I. Using the direct method, and model based and empirical temperature relations described in detail in $\mathrm{H} 08$, we have estimated the electron densities and temperatures, ionic and total chemical abundances for different atomic species. This analysis was done on the global measurements performed to the emission lines of the spectra obtained with both instruments, and on the different kinematical components deconvolved in the emission-line profiles in the high-resolution data.

For all observed knots, and for all the global measurements and kinematical components, the electron densities were found to be well below the critical density for collisional de-excitation, as is well known to occur in the star-forming processes belonging to $\mathrm{H}$ II galaxies.

From the single dispersion spectra, in knot B we measured four line temperatures: $T_{\mathrm{e}}\left(\left[\mathrm{O}_{\mathrm{III}}\right]\right), T_{\mathrm{e}}\left(\left[\mathrm{O}_{\mathrm{II}}\right]\right), T_{\mathrm{e}}\left(\left[\mathrm{S}_{\mathrm{III}}\right]\right)$ and $T_{\mathrm{e}}\left(\left[\mathrm{S}_{\mathrm{II}}\right]\right)$ reaching high precision, with rms fractional errors of the order of 2 , 3,10 and 14 per cent, respectively. For knot $\mathrm{C}$, from model-based and empirical temperature relations, we estimated the electron temperatures $T_{\mathrm{e}}([\mathrm{O} \mathrm{III}]), T_{\mathrm{e}}([\mathrm{O} \mathrm{II}]), T_{\mathrm{e}}\left(\left[\mathrm{S}_{\mathrm{III}}\right]\right), T_{\mathrm{e}}\left(\left[\mathrm{S}_{\mathrm{II}}\right]\right)$ and $T_{\mathrm{e}}([\mathrm{N}$ II] $)$, although in these cases the quoted errors for the line temperatures derived using model-based relationships are only formal errors calculated from the measured line intensity errors applying an error propagation formula, without assigning any error to the temperature calibration itself.

From the echelle spectra of knot B, we measured $T_{\mathrm{e}}([\mathrm{O}$ III $])$ and $T_{\mathrm{e}}$ ([S III]) applying the direct method. We have reached high precision from the global measure and the broad component for the first temperature, with rms fractional errors of the order of 5 and 4 per cent, respectively, and slightly worse, 15 per cent for the narrow component. We have also obtained good precision for the estimates of the [S III] temperature, 13, 18 and 14 per cent from the global measure, the narrow and the broad components, respectively. This is the first time that physical conditions are directly estimated for kinematical components of $\mathrm{H}$ II galaxies.

Using the estimated values for the electron densities and temperatures and a careful and realistic treatment of observational errors, we have estimated ionic and total abundances of $\mathrm{O}, \mathrm{S}, \mathrm{N}, \mathrm{Ne}, \mathrm{Ar}$, $\mathrm{Fe}$ and $\mathrm{He}$. For the echelle data, we have also been able to carry out a chemodynamics analysis applying the direct method to the kinematical component decomposition of the emission-line profiles. This kind of analysis had never been done until now for this type of objects. The obtained total abundances of $\mathrm{O}, \mathrm{S}, \mathrm{N}, \mathrm{Ne}$ and $\mathrm{Ar}$ are in the typical range found for $\mathrm{H}$ II galaxies. The total oxygen abundance derived for knot B is in very good agreement with the value estimated by López-Sánchez \& Esteban (2009b). The total oxygen abundances derived for all the measurements of the echelle data of this knot are in good agreement. For knot A, the derived total oxygen abundance from the global measure is lower $(\sim 0.2 \mathrm{dex})$ than the value found by López-Sánchez \& Esteban (2009b). However, taking into account all the possible sources of uncertainties we find this difference negligible within the errors. Total oxygen abundances derived for knots $\mathrm{A}, \mathrm{B}, \mathrm{C}$ and $\mathrm{E}$ are very similar among themselves considering the observational errors. The N/O ratios derived from the different components of knot A and the global measure of knot $\mathrm{E}$ are in very good agreement and show an excess with respect to the typical values found for $\mathrm{H}$ II galaxies. Knot $\mathrm{B}$ seems to also show an excess in the N/O ratio, but it is not as evident as in the case of knot $\mathrm{A}$. For knots $\mathrm{A}$ and $\mathrm{B}$, the relative abundances of N/O, S/O, Ne/O and $\mathrm{Ar} / \mathrm{O}$ for the different kinematical components are very similar; these could be evidence for a common or very similar chemical evolution for the different kinematical components of each knot. It could also be indicative that the different kinematical components are different phases of the same gas.

The ionization structure of knots A and B mapped through the use of the $\eta$ and $\eta^{\prime}$ diagrams shows very similar values within the errors for the different components of each of these regions. Only the broad component of knot A seems to have slightly higher effective temperature (lower value of the parameters) than the other components of this knot, showing similar values to those of knot B. These similarities between the ionization structure of the different kinematical components imply that the effective temperatures of the ionizing radiation fields are very similar for all the different kinematical components, in spite of some small differences in the ionization state of different elements. The ionizing star clusters that excite the gas belonging to each star-forming knot that produces the different kinematical components could therefore be the same. The difference in the ionizing structure of these two knots suggests a different evolutionary stage, with knot $\mathrm{A}$ located in a region with lower effective temperature than that where knot B is placed. This is in agreement with the presence of an older and more evolved stellar population in knot A than in knot B, as suggested by López-Sánchez \& Esteban (2010a).

We have also derived the total oxygen abundances using several strong-line empirical parameters. In general, the estimated abundances are consistent with the derived abundances using the temperatures calculated by the direct method and/or by model-based and empirical temperature relationships. We have to emphasize that all the strong-line empirical parameters were derived from measurements of the total intensity of the emission lines from entire regions, and an analysis or calibration for kinematical components has never been done. Observations with high spectral resolution and much better $\mathrm{S} / \mathrm{N}$, in particular to measure the auroral $\mathrm{O}^{+}$emission lines, are needed to develop the empirical relations for the different kinematical components.

\section{ACKNOWLEDGMENTS}

We acknowledge fruitful discussions with Enrique Pérez-Montero. We thank very much an anonymous referee for a thorough reading of the manuscript and for suggestions that greatly improved its clarity. We are grateful to the director and staff of LCO for technical assistance and warm hospitality. This research has made use of the NASA/IPAC Extragalactic Database (NED) which is operated by the Jet Propulsion Laboratory, California Institute of Technology, under contract with the National Aeronautics and Space Administration. Support from the Spanish Ministerio de Educación y Ciencia 
(AYA2007-67965-C03-03, AYA2010-21887-C04-03), and partial support from the Comunidad de Madrid under grant S2009/ESP1496 (ASTROMADRID), is acknowledged. VF and GB would like to thank the hospitality of the Astrophysics Group of the UAM during the completion of this work.

\section{REFERENCES}

Allende Prieto C., Lambert D. L., Asplund M., 2001, ApJ, 556, L63 Alloin D., Collin-Souffrin S., Joly M., Vigroux L., 1979, A\&A, 78, 200 Amorín R. O., Pérez-Montero E., Vílchez J. M., 2010, ApJ, 715, L128 Asplund M., Grevesse N., Sauval A. J., 2005, in Barnes T. G., III, Bash F. N., eds, ASP Conf. Ser. Vol. 336, The Solar Chemical Composition. Astron. Soc. Pac., San Francisco, p. 25

Barker T., 1980, ApJ, 240, 99

Benjamin R. A., Skillman E. D., Smits D. P., 1999, ApJ, 514, 307

Bohlin R. C., Dickinson M. E., Calzetti D., 2001, ApJ, 122, 2118

Cairós L. M., Vílchez J. M., González Pérez J. N., Iglesias-Páramo J., Caon N., 2001, ApJS, 133, 321

Cairós L. M., Caon N., Zurita C., Kehrig C., Weilbacher P., Roth M., 2009, A\&A, 507, 1291

Cairós L. M., Caon N., Zurita C., Kehrig C., Roth M., Weilbacher P., 2010, A\&A, 520, A90

Cumming R. J., Fathi K., Östlin G., Marquart T., Márquez I., Masegosa J., Bergvall N., Amram P., 2008, A\&A, 479, 725

De Robertis M. M., Dufour R. J., Hunt R. W., 1987, J. R. Astron. Soc. Canada, 81, 195

de Vaucouleurs G., de Vaucouleurs A., Corwin H. G., Buta R. J., Paturel G., Fouque P., 1991, Third Reference Catalogue of Bright Galaxies, version 3.9. Springer-Verlag, Berlin

Denicoló G., Terlevich R., Terlevich E., 2002, MNRAS, 330, 69

Díaz A. I., 1988, MNRAS, 231, 57

Díaz A. I., Pérez-Montero E., 2000, MNRAS, 312, 130

Díaz A. I., Terlevich E., Castellanos M., Hägele G. F., 2007, MNRAS, 382, 251 (D07)

Esteban C., Vílchez J. M., 1992, ApJ, 390, 536

Ferland G. J., Korista K. T., Verner D. A., Ferguson J. W., Kingdon J. B., Verner E. M., 1998, PASP, 110, 761

Firpo V., 2011, PhD thesis, Universidad Nacional de La Plata

Firpo V., Bosch G., Morrell N., 2005, MNRAS, 356, 1357

Firpo V., Bosch G., Hägele G. F., Morrell N., 2010, MNRAS, 406, 1094

Firpo V., Bosch G., Hägele G. F., Díaz A. I., Morrell N., 2011, MNRAS, 414, 3288 (Paper I)

French H. B., 1980, ApJ, 240, 41

García-Benito R., 2009, PhD thesis, Universidad Autonóma de Madrid

García-Benito R. et al., 2010, MNRAS, 408, 2234

García-Lorenzo B., Cairós L. M., Caon N., Monreal Ibero A., Kehrig C., 2008, ApJ, 677, 201

Garnett D. R., 1992, AJ, 103, 1330

González-Delgado R. M. et al., 1994, ApJ, 437, 239

Grevesse N., Sauval A. J., 1998, Space Sci. Rev., 85, 161

Hägele G. F., 2008, PhD thesis, Universidad Autónoma de Madrid

Hägele G. F., Pérez-Montero E., Díaz A. I., Terlevich E., Terlevich R., 2006, MNRAS, 372, 293 (H06)

Hägele G. F., Díaz A. I., Cardaci M. V., Terlevich E., Terlevich R., 2007, MNRAS, 378, 163

Hägele G. F., Díaz A. I., Terlevich E., Terlevich R., Pérez-Montero E., Cardaci M. V., 2008, MNRAS, 383, 209 (H08)

Hägele G. F., Díaz A. I., Cardaci M. V., Terlevich E., Terlevich R., 2009, MNRAS, 396, 2295

Hägele G. F., Díaz A. I., Cardaci M. V., Terlevich E., Terlevich R., 2010, MNRAS, 402, 1005

Hägele G. F., García-Benito R., Pérez-Montero E., Díaz Á. I., Cardaci M. V., Firpo V., Terlevich E., Terlevich R., 2011, MNRAS, 414, $272(\mathrm{H} 11)$
Holweger H., 2001, in Wimmer-Schweingruber R. F., ed., AIP Conf. Ser. Vol. 598, Photospheric Abundances: Problems, Updates, Implications. Am. Inst. Phys., New York, p. 23

Hoyos C., Díaz A. I., 2006, MNRAS, 365, 454

Hoyos C., Guzmán R., Bershady M. A., Koo D. C., Díaz A. I., 2004, AJ, 128,1541

Izotov Y. I., Thuan T. X., Lipovetsky V. A., 1994, ApJ, 435, 647

Izotov Y. I., Stasińska G., Guseva N. G., Thuan T. X., 2004, A\&A, 415, 87

James B. L., Tsamis Y. G., Barlow M. J., Westmoquette M. S., Walsh J. R., Cuisinier F., Exter K. M., 2009, MNRAS, 398, 2

Kehrig C., Vílchez J. M., Sánchez S. F., Telles E., Pérez-Montero E., MartínGordón D., 2008, A\&A, 477, 813

Kingdon J., Ferland G. J., 1995, ApJ, 442, 714

Kunth D., Östlin G., 2000, A\&AR, 10, 1

Kunth D., Sargent W. L. W., 1983, ApJ, 273, 81

López-Sánchez A. R., 2010, A\&A, 521, A63

López-Sánchez A. R., Esteban C., 2009a, Ap\&SS, 324, 355

López-Sánchez A. R., Esteban C., 2009b, A\&A, 508, 615

López-Sánchez A. R., Esteban C., 2010a, A\&A, 516, A104

López-Sánchez A. R., Esteban C., 2010b, A\&A, 517, A85

López-Sánchez A. R., Mesa-Delgado A., López-Martín L., Esteban C., 2011, MNRAS, 411, 2076

McGaugh S. S., 1991, ApJ, 380, 140

Miller J. S., Mathews W. G., 1972, ApJ, 172, 593

Monreal Ibero A., Vílchez J. M., Walsh J. R., Muñoz-Tuñón C., 2010, A\&A, $517, \mathrm{~A} 27$

Monreal Ibero A., Relaño M., Kehrig C., Pérez-Montero E., Vílchez J. M., Kelz A., Roth M. M., Streicher O., 2011, MNRAS, 413, 2242

Olive K. A., Skillman E. D., 2001, New Astron., 6, 119

Olive K. A., Skillman E. D., 2004, ApJ, 617, 29

Osterbrock D. E., 1989, Astrophysics of Gaseous Nebulae and Active Galactic Nuclei. University Science Books, Mill Valley, CA

Östlin G., 1998, PhD thesis, Uppsala University

Pagel B. E. J., Edmunds M. G., Blackwell D. E., Chun M. S., Smith G., 1979, MNRAS, 189, 95

Peimbert M., Costero R., 1969, Bol. Obs. Tonantzintla Tacubaya, 5, 3

Pérez-Gallego J. et al., 2010, MNRAS, 402, 1397

Pérez-Montero E., Díaz A. I., 2003, MNRAS, 346, 105

Pérez-Montero E., Díaz A. I., 2005, MNRAS, 361, 1063

Pérez-Montero E., Díaz A. I., Vílchez J. M., Kehrig C., 2006, A\&A, 449, 193

Pérez-Montero E., Hägele G. F., Contini T., Díaz A. I., 2007, MNRAS, 381, 125

Pérez-Montero E., García-Benito R., Díaz A. I., Pérez E., Kehrig C., 2009, A\&A, 497, 53

Pérez-Montero E., García-Benito R., Hägele G. F., Díaz A. I., 2010, MNRAS, 404, 2037

Pérez-Montero E. et al., 2011, A\&A, 532, A141

Pettini M., Pagel B. E. J., 2004, MNRAS, 348, L59

Pilyugin L. S., 2007, MNRAS, 375, 685 (P07)

Relaño M., Monreal Ibero A., Vílchez J. M., Kennicutt R. C., 2010, MNRAS, 402, 1635

Rodríguez M., Rubin R. H., 2004, in Duc P.-A., Braine J., Brinks E., eds, IAU Symp. Vol. 217, Recycling Intergalactic and Interstellar Matter. Astron. Soc. Pac., San Francisco, p. 188

Rosales-Ortega F. F., Kennicutt R. C., Sánchez S. F., Díaz A. I., Pasquali A., Johnson B. D., Hao C. N., 2010, MNRAS, 405, 735

Rosales-Ortega F. F., Díaz A. I., Kennicutt R. C., Sánchez S. F., 2011, MNRAS, 415, 2439

Sánchez S. F., Rosales-Ortega F. F., Kennicutt R. C., Johnson B. D., Diaz A. I., Pasquali A., Hao C. N., 2011, MNRAS, 410, 313

Sargent W. L. W., Searle L., 1970, ApJ, 162, L155

Shaw R. A., Dufour R. J., 1995, PASP, 107, 896

Shi F., Kong X., Li C., Cheng F. Z., 2005, A\&A, 437, 849

Stasińska G., 1978, A\&A, 66, 257

Stasińska G., 2006, A\&A, 454, L127

Storchi-Bergmann T., Calzetti D., Kinney A. L., 1994, ApJ, 429, 572 
Storey P. J., Hummer D. G., 1995, MNRAS, 272, 41

Tenorio-Tagle G., Munoz-Tunon C., Cid-Fernandes R., 1996, ApJ, 456, 264

Terlevich R., Melnick J., Masegosa J., Moles M., Copetti M. V. F., 1991, A\&AS, 91, 285

Terlevich R., Silich S., Rosa-González D., Terlevich E., 2004, MNRAS, 348,1191
Vílchez J. M., Esteban C., 1996, MNRAS, 280, 720

Vílchez J. M., Pagel B. E. J., 1988, MNRAS, 231, 257

Westmoquette M. S., Gallagher J. S., Smith L. J., Trancho G., Bastian N., Konstantopoulos I. S., 2009, ApJ, 706, 1571

This paper has been typeset from a $\mathrm{T}_{\mathrm{E}} \mathrm{X} / \mathrm{LAT}_{\mathrm{E}} \mathrm{X}$ file prepared by the author. 